Exploring Perceptions and Categorization of Virginia Hard Ciders Through the Application of Sorting Tasks

J'Nai Kessinger

Thesis submitted to the faculty of the Virginia Polytechnic Institute and State University in partial fulfillment of the requirements for the degree of

Master of Science in Life Sciences

In

Food Science and Technology

Jacob Lahne, Co-Chair

Amanda Stewart, Co-Chair

Clinton Neill

Thursday, December 5, 2019

Blacksburg, Virginia

Keywords: hard cider, rapid sensory analysis, packaging, consumer perceptions 


\title{
Exploring Perceptions and Categorization of Virginia Hard Ciders Through the Application of Sorting Tasks
}

J'Nai Kessinger

\begin{abstract}
Hard cider is an alcoholic beverage made from fermented apple juice. Its popularity has grown rapidly since the early 2000 s and is expected to grow to a billiondollar industry by 2022 . However, unlike beer and wine, there are few popular resources and little scholarly research on the sensory attributes of ciders and how consumers perceive them. Thus, the purpose of this study was to categorize and describe the sensory and visual product attributes of ciders made in Virginia, USA using a rapid sensory evaluation method with untrained panelists known as a free sorting task. Specifically, panelists $(N=65)$ first evaluated, sorted into groups, and described ciders $(K=18)$. Then panelists $(N=63)$ sorted photo sheets of cider labels and packaging according to how they expected the products would taste and at what occasion they would be most inclined to drink each cider. The data were analyzed with DISTATIS to produce compromise similarity maps, with bootstrapped confidence intervals to identify significant differences between products. Classical text analysis was used to evaluate the sensory descriptions used by assessors during the sorting task and project terms onto the similarity map. Panelists identified and described distinct sensory styles and attributes among the ciders evaluated. Consistent patterns in what occasion panelists might consume a cider emerged, providing a first-look into how cider might be valued based on packaging and label.
\end{abstract}




\section{Exploring Perceptions and Categorization of Virginia Hard Ciders Through the Application of Sorting Tasks}

J'Nai Kessinger

GENERAL AUDIENCE ABSTRACT

Hard cider is a beverage made from fermented apple juice. Its popularity has grown rapidly since the early 2000 s and is expected to grow to a billion-dollar industry by 2022 . However, there are few popular resources and little scholarly research on the sensory attributes of ciders and how consumers perceive them. The purpose of this study was to categorize and describe the sensory and visual product attributes of ciders made in Virginia using sorting tasks with untrained panelists. Specifically, panelists $(N=65)$ first evaluated, sorted into groups, and described ciders $(K=18)$. Panelists $(N=63)$ then sorted photo representations of cider labels and packaging according to how they expected the products would taste and at what occasion they would be most inclined to drink each cider. The data were analyzed with DISTATIS, an extension of multidimensional scaling, to produce product similarity maps with confidence intervals to identify significant differences between products. Classical text analysis was used to evaluate the sensory descriptions used by assessors during the sorting task and project terms onto the product maps. Panelists identified and described distinct sensory styles and attributes among the ciders evaluated. Consistent patterns in what occasion panelists might consume a cider emerged, providing a first look into how cider might be valued based on packaging and label. The findings act as a first step in understanding how consumers may describe and perceive hard cider and will aid in future sensory research on consumer liking, purchase intent, and acceptance of hard cider. 


\section{Acknowledgements}

I would like to express my sincere gratitude to my advisors, Drs. Jacob Lahne and Amanda Stewart, for their guidance and support throughout my time here at Virginia Tech. I would like to thank my committee member, Dr. Clinton Neill, for providing their expertise throughout the development and execution of this project. I would like to thank Grace Earnhart for being a wonderful undergraduate researcher and friend. I would like to thank Katherine Phetxumphou for all her hard work in the VT sensory lab and for being an amazing mentor. I would like to thank Leah Hamilton for her immense role in performing data analysis. Finally, I would like to thank my fellow graduate students, husband, family, and friends for all their support and encouragement since day one. 


\section{Table of Contents}

Chapter 1: Introduction and Justification............................................................... 1

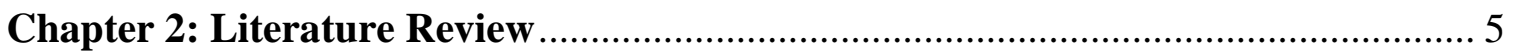

2.1. History, Definitions, and Current Cider Trends.................................................... 5

2.2. Research Framework of Consumer-Focused Sensory Evaluation of Ciders ........... 8

2.3. The Impact of Apple Cultivars, Processing and Fermentation Parameters, and

Cider Chemistry on the Sensory Attributes of Cider ................................................... 13

2.4. Descriptive Languages, Flavor Wheels, and Expertise in Beer, Wine, and Cider

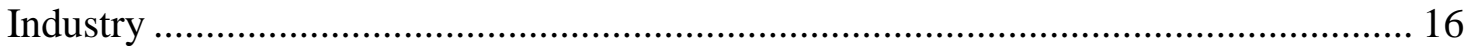

2.5. Impact of Product Packaging on Consumer Perceptions and Expectations of Wine,

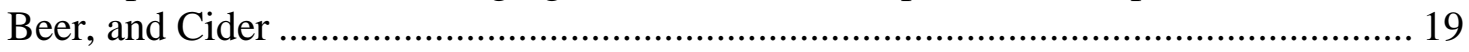

2.6. Sorting Tasks in the Sensory Evaluation of Wine, Beer, and Cider ...................... 21

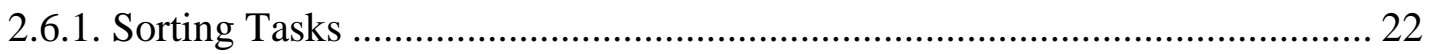

2.6.2. DISTATIS, Hierarchical Cluster Analysis, and Textual Analysis ................. 23

2.6.2. Application of Sorting Studies in the Wine and Beer Industry ..................... 26

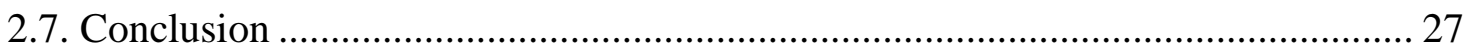

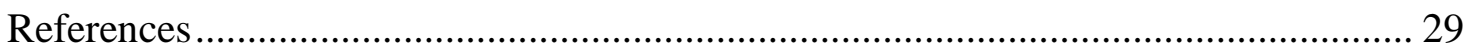

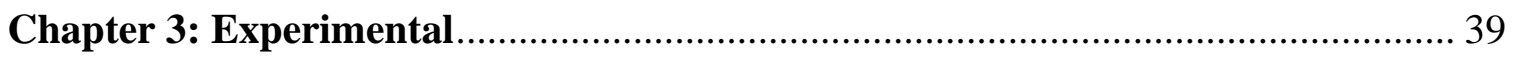

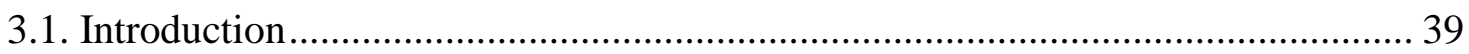

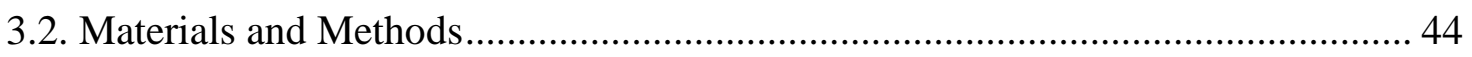

3.2.1. Study 1: Free Sorting of Ciders Through Aroma and Flavor Analysis .......... 44

3.2.2. Free Sorting of Ciders Through Visual Evaluation of Cider Packaging and

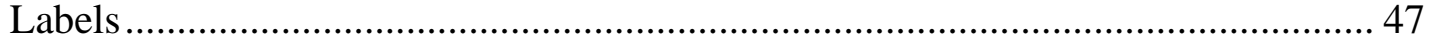

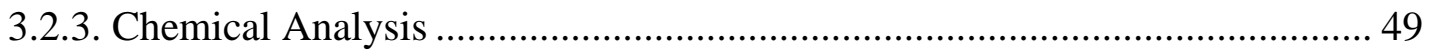

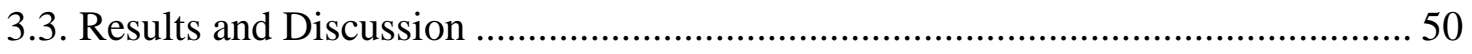

3.3.1. Demographic Survey and Questionnaire ………......................................... 50

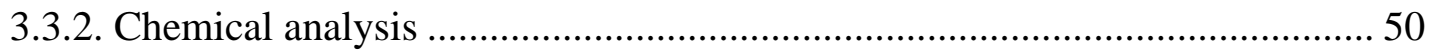

3.3.3. Study 1: Flavor and Aroma Analysis of Ciders ............................................... 51

3.3.4. Study 2: Visual Evaluation of Cider Packaging and Labels ........................... 52

3.3.5. Comparison of Producer Descriptors and Panelist Expectations and

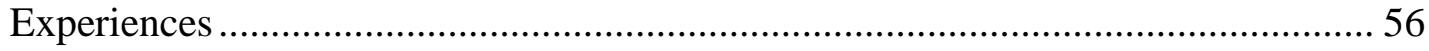

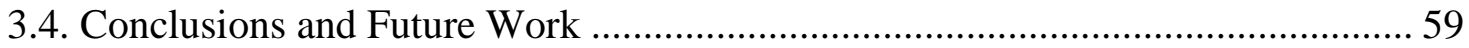

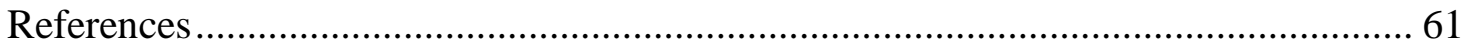

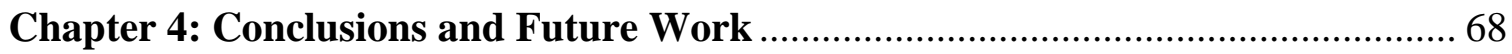

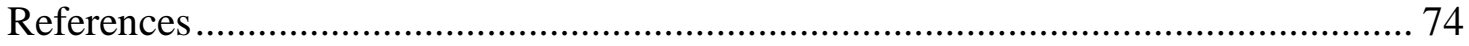




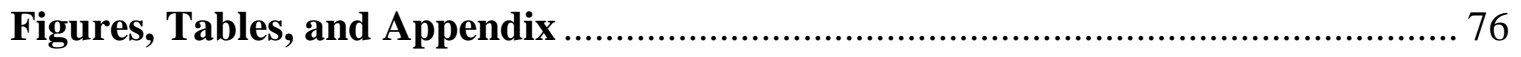

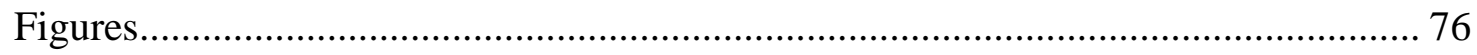

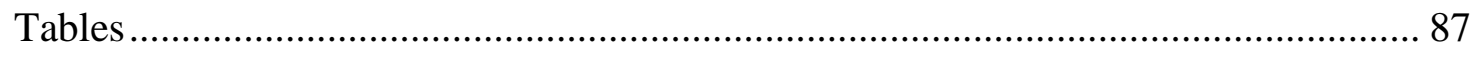

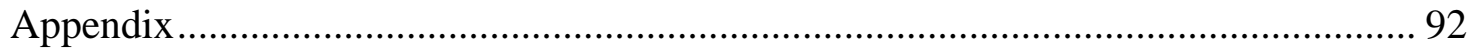




\section{Chapter 1: Introduction and Justification}

In the United States (US), the term "hard cider" refers to an alcoholic beverage made from fermented apple juice and "cider" by itself refers to unpasteurized and unfiltered apple juice. In European countries like the United Kingdom (UK), France, and Spain, hard cider is simply referred to as "cider", which is how it will be discussed throughout this paper (Lea \& Drilleau, 2003). Cider consumption in the US has risen exponentially in the past decade (Brager, 2019) and sales of regionally and locally produced, or craft, ciders have increased by $39 \%$ since 2012 (The Cider Journal, 2017). As of January 2019, there were 910 cideries established in the US and cider is commercially produced in 48 states and the District of Columbia. Virginia is both a leading apple and cider producing state, ranking $6^{\text {th }}$ and $8^{\text {th }}$ in production, respectively (Cyder Market, 2019; The Virginia Apple Board, 2019).

As interest in craft ciders continues to rise, it is important to understand their sensory attributes and how consumers describe and categorize commercial ciders. However, there appears to be little scholarly resources or academic studies on the sensory attributes of cider and fewer that incorporate average or non-cider drinkers (Fabien-Ouellet \& Conner, 2018). In wine and beer, perceptions of product attributes and their contributing flavor and aroma compounds are well-documented (Meilgaard, Dalgliesh, \& Clapperton, 1979; Noble, Arnold, Buechsenstein, Leach, \& Schmidt, 1987). Additionally, there are clearly defined styles and descriptive sensory languages, or lexicons, that allow for segmentation of consumers (Lockshin \& Corsi, 2012; Nilsson, Reid, \& Lehnert, 2018). External product attributes and labels have been found to help set expectations and consumers are more likely to re-purchase a product with which they had a positive experience (Lockshin \& Corsi, 2012; Rivaroli, Baldi, \& Spadoni, 2020). 
Professional cider organizations, like the United States Association of Cider Makers (USACM), and competitions, like the Great Lakes International Cider and Perry Competition (GLINTCAP), have developed style guides that categorize and describe ciders but there are differences in how cider styles are determined and the sensory attributes of interest. For example, the GLINTCAP style guide uses the USACM definitions as its backbone, but in addition to profiling cider styles' aroma/flavor, appearance, and apple varieties, GLINTCAP includes body, mouthfeel, overall impressions, and comments. It is also unclear if cider makers or consumers are aware of or agree with these guidelines, and cider makers have expressed an interest in establishing a 'nationally-recognized consumer-focused cider lexicon with the explicit goal of helping consumers of differing cider knowledge identify cider styles and products they are most likely to enjoy" (Fabien-Ouellet \& Conner, 2018, p. 54). In previous research, expert tasters have been found to use descriptive terminology significantly different from novices, meaning that the current style guides and descriptions may not resonate with average or non-cider drinkers (Melcher \& Schooler, 1996). To address this gap in knowledge, it is essential to begin incorporating consumer-focused sensory evaluation to create foundational knowledge of cider styles and sensory attributes to understand what makes cider such a highly sought-after commodity.

Sensory evaluation is defined as a scientific discipline that relies on human responses and is "used to evoke, measure, analyze, and interpret those responses to products as perceived through the senses of sight, smell touch, taste, and hearing" (Lawless \& Heymann, 2010, p. 2). It can be useful in profiling a product's sensory attributes and identifying and quantifying key drivers of product liking and acceptance. It is can also be used as a tool that can extrapolate how a products' experienced sensory properties match the expectations set by external attributes like 
packaging type and material, label design and color, or imagery. A sensory method that can be useful in profiling and categorizing a product's sensory attributes is descriptive analysis. This method consists of a sensory panel consisting of 8 to 12 panelists that are trained, with reference standards, to generate a consensus on the meaning of attributes of interest. During the training phase, panelists are either provided with a predetermined sensory language or taught to develop their own, a daily process that can take several weeks to perform and requires constant calibration. There are several different descriptive analysis methods and trained panelists are used to produce consistent and reproducible results that can be useful in quality control and new product development, but it can be a costly and time-consuming process (Lawless \& Heymann, 2010). Rapid descriptive analysis methods, like sorting tasks, allow for untrained panelists to quickly and cost-effectively assess relatively unexplored product spaces. Paired with textual descriptors generated by panelists, the results can be useful in identifying categories within products and guiding product development, quality assurance, and marketing (Varela \& Ares, 2014).

To begin closing the gap in sensory knowledge of cider, a preliminary study was conducted with 18 craft ciders produced in Virginia. The overall goal of this project was to profile the sensory attributes of ciders using untrained consumers with a two-part sensory study. First, panelists performed a sorting task based on the evaluation of the ciders' aroma and flavor without visual cues of color, carbonation, packaging, label, and brand. Then, panelists were asked to visually evaluate photos of the ciders' packaging and labels and perform two separate sorting tasks based on 1) how they expected them to taste and 2) what occasion they might consume them in. $\mathrm{pH}$, residual sugars (glucose and fructose), and titratable acidity (malic acid 
equivalents) were measured in analytical triplicate to allow for a preliminary catalog of the physicochemical composition of Virginia craft ciders.

The results of this study include 1) a sensory profile of Virginia craft ciders, 2) the influence of cider packaging and label on anticipated sensory attributes, 3) the influence of cider packaging and label on occasion-based consumption, and 4) a comparison of producer-generated sensory descriptions, consumer expectations, and consumers' sensory experience. As cider is a relatively unexplored product space with little understanding of consumers' sensory expectations and experiences, these findings can be used to begin defining the sensory language consumers use when evaluating and discussing the visual and sensory attributes of products. These results can be used as a foundational framework in future sensory work and assess consumer liking, preference, acceptance, and purchase intent, revealing the importance and valuation of cider and their attributes through the consumers' perspective. This knowledge may be useful increasing access to knowledge to small- and medium-sized cider producers that might be useful in understanding their consumers, the development of best practices, and understanding if sensory changes have occurred after bottling during transport, distribution, and storage. 


\section{Chapter 2: Literature Review}

\subsection{History, Definitions, and Current Cider Trends}

Cider was once considered "America's drink" due to its overwhelming popularity from the conception of the nation (Malone-Brown, 2014). It came to the US alongside European colonists in the 1600s and quickly became the most consumed and popular beverage; in the 1790s, cider consumption per capita of the population aged 15 and over was 34 gallons a year (Rorabaugh, 1976). However, cider's popularity diminished in the late-1800s due to the rise of the Industrial Revolution and Prohibition. There were fewer farmers to tend to their crops and Prohibition regulations saw to it that the majority of cider orchards were destroyed. Heirloom cider apple cultivars were scarce and the majority of apple crops consisted of eating apples. The influx of German immigrants and their expertise in ale and beer making crushed cider as America's favorite beverage (Malone-Brown, 2014; Rupp, 2015).

The tradition of cider making has been kept alive by small-scale farmers and enthusiasts (WSU Extension, 2019). As interest in artisanal and traditional products increases, so has interest in cider. This interest is reflected in an explosive growth in overall and craft cider sales and a number of established cideries during the past ten years. As of January 2019, there were 910 cideries established in the US compared to the 94 in 2008. Cider is commercially produced in 48 states and the District of Columbia (Benivia, LLC, 2019). In 2008, cider only made up $0.06 \%$ of the alcoholic beverage industry, with sales totaling $\$ 44$ million (Nielsen, 2018; Nurin, 2018). In 2018, cider made up $0.4 \%$ of the alcoholic beverage industry and sales reached $\$ 506$ million (Nielsen, 2018; Nurin, 2018).

Though ciders that are produced by larger companies such as Strongbow (Heineken), Angry Orchard (The Boston Beer Company), and Crispin (MillerCoors) have been a significant 
contributor to overall cider sales, "craft ciders" or ciders made by regional or local producers have contributed greatly to the resurgence in cider sales (Brager, 2019). 4

This interest is attributed to consumers, especially those who are younger, looking to experience "unique products with their own signature style and taste" and seeking "alternatives to mainstream products" (Thériault, 2019). In a 2016 survey conducted by Nielsen for the United States Association of Cider Makers, $65 \%$ of surveyed consumers agreed or strongly agreed that they prefer to purchase from local cideries and $68 \%$ of surveyed consumers agreed or strongly agreed that, lately, they had been purchasing more craft cider. These attitudes can potentially explain the expansion of the craft cider segment, with products selling at premium prices compared to large commercial ciders, beer, and flavored malt beverages (Crompton \& Brager, 2017; The Cider Journal, 2017).

Though cider sales are high and continued growth is anticipated, cider makes up less than $1 \%$ of the alcoholic beverage market share. There is little research on differentiating cider styles and the demand for cider made with specific apple cultivars. Where and how cider is produced and the sensory characteristics it contains are not clearly defined, producing products that are described inconsistently throughout the cider industry (Fabien-Ouellet \& Conner, 2018). There also appears to be a gap in knowledge in what the general public knows and understands of cider. In the US, non-cider drinkers don't necessarily know what cider is, where it comes from, or how it's made and there are opportunities to grow the cider industry through education, marketing, and advertising (Fabien-Ouellet \& Conner, 2018; Thériault, 2019). This lack of knowledge may help explain the small market share. In a 2017 web-based poll conducted by the Angry Orchard cider brand, $37 \%$ of adults aged 25 - 49 were unable to name a cider brand. Of those who did name a brand, 9.3\% named Angry Orchard; 6.9\% named Mike's Hard Lemonade, a flavored 
malted beverage; and 6.1\% named Redd's Apple, which is another flavored malted beverage (Bernot, 2017).

The wine and beer industry has demonstrated that products with clear and defined styles have a positive influence on repeat purchase, brand loyalty, and recommendation amongst consumers. In a literature review conducted by Aquilani et al. (2015), factors that have been found to contribute to consumers' beer choice and consumption are price, brand, production differentiation (craft versus commercial beers), and packaging. The success of craft beer is thought to be due to its quality, availability on the market, competitive pricing, and its differentiation to commercially available beer. A literature review performed by Lockshin \& Corsi (2012) found that consumers' wine involvement and sensory preferences along with price, packaging, brand, and origin are important in purchasing and consumption of wines. Compared to beer or wine, there are no well-defined standards for categorizing cider styles, and with such a wide array of cider styles and brands, consumers are "faced with a difficult task of making a cider purchase based on inconsistent information on the product label" (Tozer, Galinato, Ross, Miles, \& McCluskey, 2015, p. 315).

There are currently available cider style guides that have been developed by professional organizations like the United States Cider Maker Association (USACM), the Great Lakes International Cider Perry Competition (GLINTCAP), and CIDERCRAFT Magazine. It is unclear how these guides are presented to and used by consumers of varying knowledge, cider makers, and cider professionals Additionally, how ciders are categorized and described is organizationdependent. For example, the CIDERCRAFT tasting guide characterizes ice ciders as a dessert cider while the USACM considers it a category all on its own. The GLINTCAP style guide builds on the USACM style guide but has additional sensory attributes of interest such as 
mouthfeel or overall impression. Another potential issue with professional-generated terminology is that many studies have identified significant differences between the terminology used by trained assessors, product experts, and novice tasters (Hopfer \& Heymann, 2014; Lelièvre, Chollet, Abdi, \& Valentin, 2008; Melcher \& Schooler, 1996). Thus, there are opportunities to define cider styles and categories with a descriptive sensory language, or lexicon, that can be used universally by both non-cider drinkers and cider experts during selection, consumption, and discussion. Further research in this area should expand to research and collaboration with both cider experts and average and non-cider consumers to gauge how a universal cider lexicon might be developed and applied.

As interest in regional and local ciders increases, there are opportunities to identify if they possess sensory attributes consumers can categorize and describe. Profiling ciders' sensory attributes can be incorporated in the foundational knowledge necessary in the development of a more universal and representative descriptive cider language. By defining what makes these ciders unique, cider makers may be able to identify what consumers like, create resources for cider sales teams, and identifying what attributes from the cidermaking process are most and least desirable.

\subsection{Research Framework of Consumer-Focused Sensory Evaluation of Ciders}

Much of the early research conducted on ciders can be traced back to the Long Ashton Research Station (LARS). Established in the 1890s, what began as a series of experiments on cider making turned into the establishment of the Department of Agricultural and Horticultural Research of the University of Bristol. During the 1970s and 1980s, examples of research conducted on cider include working to define the current framework of flavor research and the 
cider industry (A. A. Williams, 1974), formulating sensory, physical, and analytical methods to assess the quality of apples, apple juice, and cider (Lea \& Arnold, 1978; A. A. Williams, Lea, \& Timberlake, 1977), and the development of a descriptive vocabulary and profiling method for cider and perry aroma constituents through gas chromatography (GC) and sensory evaluation (A.A. Williams, 1975). Williams emphasizes that pairing flavor chemistry and sensory evaluation provides a richer, more detailed depiction of the role of flavor and aroma compounds on sensory experiences. This research provides much of the current framework of cider flavor chemistry but a large body of work conducted at the Pomology and Food and Beverages Division of LARS was concluded or left unfinished upon its closure in 1981 (Anderson, 2002).

In cider research, sensory analysis is most often used as a tool to identify how processing, storage conditions, volatiles, or cider composition may influence the sensory attributes of ciders. Few papers have a primary focus on profiling and characterizing the sensory attributes as perceived by cider consumers. Rather, trained panels of experienced assessors commonly evaluate experimental ciders' aroma and flavor in addition to indicating their liking for ciders produced under various conditions.

Another trend found in current sensory research is the primary mode of sensory evaluation is descriptive analysis (as described in chapter 1). Descriptive analysis panels were presented and trained with a predetermined vocabulary to participants rather than permitting them to develop their own. Additionally, panelists were either expert sensory evaluators or trained for the study. This appears to be done to identify either desirable or undesirable attributes of ciders with specific descriptors, a measure of quality control that is commonly accomplished with descriptive analysis. 
Quality control is a primary focus for much of the sensory research performed on cider. For example, Anton et al. (2014) pairs sensory analysis with gas chromatography-olfactometry analysis to identify the concentrations of compounds found in cider and at which concentrations they become detectable in human subjects. The goal of this study was to quantify the aromatic composition of ciders to identify compounds responsible for their potential olfactometric and sensory profiles and where they develop in the cidermaking process. Results from sensory analysis indicated that there was a significant difference among samples for the attributes of "fruity", "floral", "lactic", and "lees". Chemical analysis allowed for the identification of two volatile phenols (4-EG and 4-EP) that are responsible for the perception of the sensory attributes sweet, spicy, and lees. These findings help explain how flavors develop in the cider fermentation process, which may be a valuable tool for cidermakers to identify where to create potential quality assurance checkpoints to create a desirable product. Other examples of studies that incorporate sensory evaluation as a measure of quality control are those that aim to understand the contribution of cider's composition and flavor and aroma compounds perceived sensory attributes (Anton, Valles, Hevia, \& Lobo, 2014; Peng, Yue, \& Yuan, 2009; Symoneaux, Guichard, Le Quéré, Baron, \& Chollet, 2015), assessing how processing and storage parameters may impact the sensory attributes of cider (Lachowicz et al., 2019; Peng, Li, Cui, \& Guo, 2015; Sánchez et al., 2014; Scott \& Swaffield, 1998), and generating a predictive model evaluating the impact of apple procyanidins, fructose, acid content, and ethanol on astringency, sweetness, and sourness (Symoneaux, Chollet, et al., 2015). These studies, with the exception of Symoneaux et al. (2015) also incorporate trained and/or experienced sensory panels to assess and describe the sensory attributes of ciders produced under varying conditions. Symoneaux et al. (2015) used two separate panelists, one consisting of trained sensory assessors and another consisting of 
expert cider professionals, to validate a predictive model that aimed to use measurements and sensory contributions of malic acid, fructose, ethanol, and type and quantity of procyanidins to predict the sensory attributes of ciders based on their composition. Research performed by Qin et al. (2018) is unique to that previously mentioned sensory research as it incorporates a trained descriptive analysis panel of experienced assessors that developed their own vocabulary for evaluating commercial Scandinavian and UK ciders. Various chemical analyses were also used in tandem to profile and commercialize ciders included in this study. The rigor of the descriptive analysis panel and chemical analyses provides a large body of information on these ciders but there is little information on who the panelists are or their experience with cider. However, there are opportunities to identify if there are significant differences between the developed descriptive vocabulary and evaluations of ciders and those of average and non-cider drinkers. These studies build on the work conducted at LARS in the 1960s and 1970s and provide increased insight on the correlation of cider's chemistry processing and storage parameters on its resulting sensory attributes with advanced methodologies. In a study conducted by Lachowicz et al. (2019), the main research objective was to evaluate the influence of selected yeast strains and storage time on cider chemistry (ex: alcohol, $\mathrm{pH}$, titratable acidity), the concentration of polyphenols, and antioxidative activity on cider produced with a novel apple cultivar. Consumers were asked to hedonically rate the ciders' taste, aroma, color, and consistency. This study incorporates sensory evaluation as a means to develop a cider with desirable sensory attributes; though sensory evaluation is important in new product development, it was not used to profile the sensory attributes of the ciders developed in this study. This work aims to guide the development of best practices in cider making, but it is unclear how these ciders might be perceived by consumers. Specifically, there are opportunities to expand on this research by involving sensory assessments 
of ciders by consumers with varying cider knowledge to further understanding of how consumers may perceive, categorize, or describe ciders.

There are few studies that incorporate consumers in evaluating the sensory attributes of cider. Tozer et al. (2015) is one paper that aims to close the gap in knowledge of what is known about cider and its consumers. In this study, an untrained panel of consumers was asked to indicate their liking and willingness-to-pay (WTP) of four commercially available craft ciders. Chemical composition and consumer demographics and cider consumption preferences and habits were also collected. Their results indicate that there is a complex relationship between the measured variables, an unfamiliarity with craft cider outside of national producers, and an openness to trying craft ciders, further revealing the need for craft cider producers to raise awareness of their product to non-craft cider drinkers. Tozer et al. speculates that as consumers become increasingly familiar with craft ciders, consumers' tastes may evolve and thusly increasing WTP. They also state that the definition of "craft" may vary from consumer to consumer, producing greater ranges of WTP based on understanding.

Sugrue and Dando (2018) and Jamir et al. (2020) are very recent publications that incorporate consumer-focused sensory research. The work performed by Sugrue and Dando explore the cross-modal influence of cider color and product label on consumers' perception and expectations of the ciders' sensory attributes, giving insight into the complex role of external product attributes of cider packaging on product expectations. Jamir et al. compares crosscultural differences between the descriptive terms generated and used by Chinese and American panelists, finding that variation in how these two demographics described and categorized samples. Both studies use untrained consumers of alcoholic beverages and ask that they generate 
their own descriptive vocabulary and demonstrate the importance of understanding who cider consumers and the terminology they use in research.

\subsection{The Impact of Apple Cultivars, Processing and Fermentation Parameters, and Cider Chemistry on the Sensory Attributes of Cider}

There are thousands of cider apple cultivars that are used in cider making and can be separated into six categories based on acidity and sugar content: sweet, sour, sharp, bittersweet, bittersharp, and bitter. The blending of different apples is common and produces ciders that are balanced and have various sensory qualities (Coton \& Coton, 2016). The chemical composition of apples such as levels of sugars, acids, free amino acids, yeast assimilable nitrogen (YAN), and polyphenols has been well-documented to contribute to the aroma and flavor profile of cider (Alberti et al., 2016; Lea \& Drilleau, 2003; Ma, Neilson, Lahne, Peck, O’Keefe, \& Stewart, 2018; Rosend, Kuldjärv, Rosenvald, \& Paalme, 2019; Ye, Yue, \& Yuan, 2014). Polyphenols are responsible for many sensory attributes of cider including color, bitterness, and astringency. There also may be interactions with the sweetness and sourness of the final product and influence aroma through enzymatic decarboxylation during fermentation (Lachowicz et al., 2019). Juice processing conditions can play a role in the final nonvolatile flavor, specifically the oxidation of procyanidins. Procyanidins are a class of polyphenols known as tannins that are known to contribute to bitterness and astringency. Oxidation of procyanidins has been documented to minimize the bitterness and astringency in the final product (Lea \& Arnold, 1978; Lea \& Drilleau, 2003).

Yeasts are the primary fermenters in cider production and convert fermentable sugars to CO2, ethanol, sorbitol, and glycerol (Garai-Ibabe et al., 2008). The length of the fermentation 
and the development of compounds responsible for a product's sensory attributes are influenced by numerous factors. Nutrients available to yeasts during fermentation play a major role in their longevity. Amino acids are the main nitrogen source for yeasts during fermentation. The concentration of amino acids and the type of yeast strain can influence the sensory attributes of the final product as amino acids produce intermediates or precursors for some volatile compounds. YAN, composed of primary amino acids and ammonium ions, and its concentration in juice can affect cider fermentation rates and their deficiency can "[cause] slow or incomplete fermentation and the production of sulphur off-aromas" (Ma, Neilson, Lahne, Peck, O'Keefe, \& Stewart, 2018). Nutrients present or added to fermentation along with antimicrobials, like sulfite $\left(\mathrm{SO}_{2}\right)$, can influence the predominance of microorganisms in the natural microflora in apples or in specified starter cultures and thus the final product. Yeast proliferation can also be influenced by the levels of oxygen, sugars, and free amino acids prior to and during fermentation along with the strain's ability to tolerate ethanol and polyphenols (Lea \& Drilleau, 2003).

There are numerous compounds that are responsible for the sensory attributes found in cider. Higher alcohols, esters, fatty acids, and carbonyls are examples of important aroma compounds that are formed as secondary metabolites during fermentation (Lea \& Arnold, 1978; Qin, Petersen, \& Bredie, 2018; Rosend et al., 2019). Ethanol concentration may also affect perceptions of bitterness and astringency due to ethanol's ability to increase the solubility of tannic compounds (Lea \& Arnold, 1978). Glycerol, after ethanol, is another product of fermentation and contributes to the body of cider such as smoothness or roundness (Garai-Ibabe et al., 2008).

A cider producer may take steps to have their cider undergo malolactic fermentation. Malolactic fermentation is a secondary fermentation that decarboxylates malic to lactic acid, 
causing the sharp acidity of malic acid to fall and also contributes to the perception of ciders' being as well-rounded and more complex. Malolactic fermentation should be carefully controlled as if it progresses, D-lactic and acetic acids begin to form, which are associated with undesirable sensory attributes. (Lea \& Drilleau, 2003).

After alcoholic and malolactic fermentation, it is important to deactivate any remaining microbes that may lead to sensory changes or spoilage during aging, bottling, or storage. Specifically, the abundance of Lactobacillus species and Oenococcus oeni can metabolize residual carbon sources (fructose, glycerol, lactic acid), which could lead to acetification, ropiness, or bitterness (Garai-Ibabe et al., 2008).

There are many factors cider producers must consider during cider making that can influence their cider. Apple variety, ripeness, storage conditions, and processing are examples of prefermentation factors that can affect the final product. Decisions made during and postfermentation are also of great importance such as the length and temperature of fermentation, yeast strains, aging conditions, and addition of antimicrobial or fining agents. There are many ways through which cider makers can customize and control the production of their ciders.

Though there is current work in profiling the chemical composition of Virginia apple cultivars and their management postharvest (Ma, Neilson, Lahne, Peck, O'Keefe, \& Stewart, 2018; Thompson-Witrick et al., 2014), and identification of best practices (Boudreau, Peck, O'Keefe, \& Stewart, 2017; Ma, Neilson, Lahne, Peck, O'Keefe, Hurley, et al., 2018), there are opportunities to explore how these findings correspond with consumer perceptions of ciders made from Virginia apple cultivars through sensory analysis. 


\subsection{Descriptive Languages, Flavor Wheels, and Expertise in Beer, Wine, and}

\section{Cider Industry}

Professional cider industry organizations, such as CIDERCRAFT magazine, the United States Association of Cider Makers (USACM), and the Great Lakes International Cider Perry Competition (GLINTCAP), have garnered the expert opinions of seasoned cider tasters to create guides that discuss the variations of alcoholic ciders and their sensory properties.

Erin James of CIDERCRAFT Magazine worked with cider experts to develop a tasting guide outlining how to taste ciders and established eleven cider categories: orchard-based, modern, single-varietal, dessert, hopped, rose, fruit-infused, barrel-aged, spiced and botanical, specialty and perry. The USACM style guide splits ciders into two styles: standard and specialties. Standard styles include modern ciders and perries and heritage ciders and perries. These categories are determined by the type of apples or pears used in the cider's creation (ex: culinary or cider and perry-specific varieties), with emphasis placed on tannin content and acidity. Specialty ciders include fruit, hopped, spiced, wood-aged, sour, and ice ciders. These categories are determined by fruit source, how they ferment, non-traditional additions, or other processing differences. Each category describes the cider's expected aroma/flavor, appearance, and fruit varieties (when applicable). The USACM also announced the launch of the Cider Lexicon project in 2018 to "make it easier for consumers to find a cider they like" (USACM, 2019). In its first phase, the project consists of three major components: The already developed and available USACM cider style guide, the development of a cider vocabulary guide, and a dryness scale.

The cider vocabulary guide aims to compile a descriptive language terminology used to discuss ciders, however, the USACM states that "cider borrows some words from wine, some 
from beer, and some are unique to cider". This appears to mean that this guide will contain words used to describe wine and beer, which may help consumers recognize ciders they might enjoy but it remains unclear to how these terms are generated and how this guide will be distributed and used by the cider industry. The dryness scale is also an effort to define and unify the usage of "dry", "semi-dry", "medium", "semi-sweet", and "sweet". The USACM is awaiting the launch of the Orchard Based Dryness Scale, currently awaiting a certification process and is continuing to refine tannin thresholds for the scale, that is currently being developed in collaboration with the New York Cider Association and Cornell University (New York Cider Association, 2019; USACM, 2019). The website Cider Language (https://ciderlanguage.com) was inspired by this project. It is independent of the USACM's Cider Lexicon project and has surveyed the labels of over 500 cider labels from US cider producers from 35 states in an attempt to "provide a foundation for further definition, improvement, and unification of language across the American cider industry" (Barry, 2019). This project creates a "starting point" of the language currently in use by cider makers on the label, generating a data set that compiles the language as it is currently described by cider makers and what is currently seen by consumers.

The style guidelines developed by the judges of GLINTCAP contains the backbone of the USACM style guide and includes criteria for three styles: standard, specialty, and intensified and distilled ciders. There are extensions to the original content as well as additional subcategories and descriptors. Ciders are then split into subcategories, where they are given a brief description and must meet specified aroma, flavor, appearance, mouthfeel, and overall impression qualifiers. Standard styles include modern, heritage, traditional, natural, and rose ciders and modern and traditional perries. These categories are determined similarly to those in the USACM style guide but definitions and descriptors for not just US ciders but also English, French, and Spanish 
styles. Specialty styles include fruit, hopped, spiced, wood-aged, and wood-aged specialty ciders and specialty and unlimited cider and perries. Intensified and distilled styles include ice and fortified ciders and spirits.

It appears that each association characterizes ciders differently. For example, the CIDERCRAFT tasting guide characterizes ice ciders as a dessert cider while the USACM considers it a category all on its own. The GLINTCAP style guide is the only resource among the three that includes distilled ciders in their guides. Cider and perry styles, their categories, and descriptive terminology are all developed among experts and how these are presented to consumers is not clear among each organization. There are also varying usage of descriptive terminology depending on the style.

In the beer and wine industry, there are developed lexicons and styles used to categorize and describe products. These lexicons have been developed as flavor wheels, a circular list with categories and subcategories of descriptive terms, that allow for an easily usable version of long lists of descriptors. These lexicons are developed by experts during industry group meetings, developing categories of descriptive terms that have reference standards to increase the wheels' utility (Meilgaard et al., 1979; Noble et al., 1987). These flavor wheels are easily searchable and available in various formats free of charge. These papers emphasize the non-static nature of these flavor wheels, stating that their continuous up-keep is necessary. Flavor wheels and the lexicons they are developed from are multifaceted tools that can be used in product commercialization, to communicate easily and effectively, and to aid product development and quality control (Suwonsichon, 2019). As the usage of flavor wheels and their terminologies may differ depending on the product and sensory expertise, there have been recent academic studies that 
have updated or developed and tested more consumer-friendly lexicons to bridge this gap

(Danner et al., 2016; Mora, Giussani, Pagliarini, \& Chaya, 2019).

In direct comparison, the only flavor wheel available on cider was developed by CIDERCRAFT magazine using both Anthony A. Williams 1975 paper and the work of biochemist Andrew Lea (http://cider.org.uk). Though the work performed by both researchers provide great information on the profile and descriptive terms applied to UK ciders, the work has not been updated in the same capacity as the wine or beer industry. CIDERCRAFT magazine's flavor wheel is also not easily accessible outside of its publication, proving to be an obstacle in accessing and using a universal cider language. This tasting guide provides a vocabulary for cider novices and experts alike to use while enjoying the drink. However, the language developed may be repetitive, not transfer easily to consumers, or differ from consumer to consumer.

\subsection{Impact of Product Packaging on Consumer Perceptions and Expectations of}

\section{Wine, Beer, and Cider}

Consumers make decisions about food prior to its consumption. Packaging attributes, like material, color, and shape, and its labels, like design, font, or information, sets consumers expectations of what the sensory attributes they'll experience, how likely they are to purchase the product, and can determine how much they are willing-to-pay (WTP). How well that product sets expectations once consumed can determine consumers' re-purchase intent and recommendations (Raz et al., 2008).

Sugrue and Dando (2018) perform one of the few studies that evaluate the role of label color on sensory perceptions of ciders. In the label evaluation, panelists were first asked to inspect a 
simplistic label featuring a red or green apple prior to consuming the provided cider sample. They then were asked to rate the label's appeal and fit-for-concept for the cider. The results were then compared to control (no label). It was found that while consumers did not prefer one label over another, there was a profound effect on their perceptions of the ciders. For example, ciders with green labels were rated to be more flavorful than the control Assessors also rated ciders with red labels as being sweeter and fruitier than control. The label also had a significant impact on the ciders' overall and flavor liking. Ciders were also rated higher in fruitiness, sweetness, apple flavor, body/mouthfeel, and "refreshingness". Sugrue and Dando speculate that the label color is more intense than that of the actual cider, creating stronger sensory responses than with just the cider alone. The role of color on expected and experienced sensory attributes has been reported in numerous studies and the label is a critical point to connect with consumers (Spence et al., 2015). This study provides a glimpse into how consumers may interact and experience a product well before it is actually consumed, knowledge that cider makers can consider and incorporate into their packaging and cider making.

In the wine and beer industry, the role of external packaging attributes has been wellreported. For wine, a systematic review performed by Lockshin \& Corsi (2012) found that label images, colors, and wine design have a positive effect on purchase intent and value. For occasions, like dinner, purchase intent can vary. Labels, images, verbiage, and statements are more important than traditional indicators of purchase intent like grape variety and words and often outperform metaphorical expressions. As there are limited studies profiling the external packaging attributes of cider, the research outlined in this paper aims to profile these attributes that may influence how cider is expected to taste and the occasion they are consumed. These 
findings will provide a first-look into how well ciders set sensory expectations and the role of packaging on occasion-based consumption.

\subsection{Sorting Tasks in the Sensory Evaluation of Wine, Beer, and Cider}

Profiling the sensory attributes of products is crucial in product research and development, quality control, and marketing. It can be useful in understanding changes in products during shelf-life or identifying relationships between raw material or processing parameters on the final product. Sensory profiling is commonly performed with a small panel of assessors are trained to identify and quantify the profile of products of interest. Conventional methods include Quantitative Descriptive Analysis (QDA), Quantitative Flavor Profiling, and the Spectrum $^{\mathrm{TM}}$ method. A commonality shared with these methods is that while training and maintaining a trained panel can be efficient, it can be a costly and time-intensive process. Panelists must first generate a list of terms that are objective, clear, unique, and independent. They are then trained to rate the intensity of each attribute, typically on a line scale. Before panelists can evaluate products, their performance is checked for repeatability, discrimination, and agreement. The data generated can provide quantifiable and reliable information on the sensory attributes of a product, but differences in project objectives can mean varying definitions of precision and sensitivity. Another barrier that arises with conventional sensory profiling methods is that the developed protocol is only applicable to a very specific set of products and is not widely applicable outside of them (Chollet, Lelièvre, Abdi, \& Valentin, 2011).

Since the 1980s, there have been efforts to establish alternative rapid sensory profiling methods that produce results similar to more conventional ones but do not require trained panels, 
making them less costly and time-intensive to perform. One example of such methods includes the sorting task (Chollet et al., 2011).

\subsubsection{Sorting Tasks}

Sorting tasks are a novel rapid sensory analysis method that collects similarity data "in which each assessor groups together stimuli based on their perceived similarities" (Varela \& Ares, 2014, p. 207). It is a simple procedure that is based on categorization and does not require a quantitative response. Free sorting tasks are completed in a single session where all products are presented simultaneously. They are displayed in a randomized order on a table with a different order for each assessor. Assessors are instructed to evaluate the sensory attributes of a set of products and then sort them into mutually exclusive groups based on their perception of product similarities. They are free to sort samples based on any criteria they chose into at least 2 groups and no more than $K-1$ groups. Sorting tasks can be extended to involve textual labeling of the groups of products assessors generate. This labeling can be performed with any criteria the assessor chooses or based on a provided written prompt. (Varela \& Ares, 2014). Statistical analysis of sorting data can reveal the structures of the product space and allow for the interpretation of its underlying dimensions (categories).

The use of a sorting task has several advantages in data collection. First, sorting tasks are a relatively simple rapid task for panelists to complete and the data it produces are representative of more intensive sensory methods. Second, groups created by assessors can assist in creating an understanding of how consumers classify and discuss cider. Third, analysis of sorting data using DISTATIS can allow for the identification of differences in ciders and individual assessors in 
addition to the ability to make statistical inferences with confidence intervals (Lahne, Collins, \& Heymann, 2016).

In traditional descriptive analysis, a panel of assessors is trained with references to understand and agree on sensory attributes used to define a product or commodity. During panel training, assessors are exposed to a wide range of food products and reference scales in a process that commonly takes several weeks of daily training sessions. Panel calibration is necessary in order to ensure consistent understanding and application of sensory attributes and their intensities among the panel. They can also be costly to maintain depending on how frequently a panel is needed (Lawless \& Heymann, 2010). Conversely, sorting tasks can be performed with untrained assessors while producing results similar to their trained counterparts. They are simple for panelists to complete in one sitting and can be used to evaluate a wide range of products. Sorting tasks are also less costly and time-intensive than traditional descriptive analysis while producing similar results. Comparatively, the data generated from sorting tasks require more in-depth statistical analysis to understand results and identify relationships among products. In this study, DISTATIS, hierarchical cluster analysis, and textual analysis were used to identify the product space of Virginia ciders and categories of products.

\subsubsection{DISTATIS, Hierarchical Cluster Analysis, and Textual Analysis}

Sorting data is that it can be analyzed in numerous ways. Multidimensional scaling analysis, multiple correspondence analysis, hierarchical cluster analysis, and DISTATIS have all been performed on sorting data to understand relationships between a series of products (Chollet et al., 2011). For this study, however, the chosen analytical methods were DISTATIS, hierarchical clustering (HCA) using Ward's method, and textual analysis. 
DISTATIS is a method of multivariate statistical analysis that is used to obtain metric distances from binary dissimilarity measures: that is, DISTATIS transforms the groupings of products from sorting into estimates of distance among these same products (Lahne et al., 2016). This method is an extension of multidimensional scaling that accounts for "individual variation of subjects' partitioning of the product space before [the] generation of a consensus solutionspace" (Lahne, Collins, \& Heymann, 2016, pp. S1264). This is done by scaling individual subject sorting matrices with double-centering and then producing a subject agreement matrix using $R_{V}$ coefficients (Lahne et al., 2016). $R_{v}$ coefficients are a measure of similarity between squared symmetric matrices and act as a means to analyze multivariate techniques such as DISTATIS (Abdi, 2007).

The product of this method is a "map' that conveys, spatially, the relationships among items, wherein similar items are located proximal to one another, and dissimilar items are located proportionately further apart” (Hout, Papesh, \& Goldinger, 2013, pp. 2). An advantage of scaling individual sorting matrices is to allow for the identification and segmentation of an assessor or set of assessors, which can identify atypicality or analysis of sets of assessors based on demographics, sorting similarity, or other criteria. Bootstrap confidence ellipses can be used to identify significant differences between products and groups. Bootstrapping is a cross-validation technique that allows for the estimation of the stability, reliability, or variability of multivariate data by resampling with replacement using the original dataset (Bruce \& Bruce, 2017). Resampling (with replacement) can be repeated a large number of times (say 1,000) and generates a simulation of the sorting data that are then projected in the form of confidence ellipses on the DISTATIS map. If the ellipses of two products do not overlap, then it can be deduced, with 95\% confidence, that they are statistically different. Conversely, if the ellipses of 
two products intersect, then it can be concluded that, with $95 \%$ confidence, they are statistically different (Abdi, Dunlop, \& Williams, 2009).

Hierarchical cluster analysis can then be used to identify groups of products that are most similar to each other. This is accomplished by first identifying the two products with the smallest distance from each other in the 2-dimensional product space (and thus most similar), clusters them, and repeats this process until all products are clustered. With Ward's method, clusters are formed so that the within-group sum of squares is minimized between each step of clustering (NCCS, n.d.).

Textual analysis is a methodology that involves understanding language and analyzing textual data to "provide a deeper understanding through the description of [the] interpretation of messages found within the text (or across texts) (Hawkins, 2018). Analyzing textual data involves using two or more coders who are familiar with the data and the research question. Preparing textual data for analysis starts with cleaning the text by removing typing mistakes, managing digits, punctuation marks, hyphens, and a case of letters. The data is then stripped of common "stop words" (ex: "to", "from", and "the") and non-descriptive verbiage before being de-stemmed (removal of endings such as affixes like "-s", “-y", or “-like”), lemmatized (grouping inflected forms together as a single base form such as combining "fruity" and "fruit"), and synonymized ("tart" and "sour" would be grouped together as "tart" based on frequency of usage by assessors) (Rinker, 2018). The selection of keywords can be determined based on a "cut-off value" or the minimum number of times a word is used by assessors. Word frequency and combinations can allow for an alternative way to construct relationships between products and sorting configurations (ten Kleij \& Musters, 2003). 


\subsubsection{Application of Sorting Studies in the Wine and Beer Industry}

Sorting tasks have been applied in the wine and beer industry with great success, which may act as a benchmark comparison for their application in researching and defining the cider category. Chollet et al (2011) investigated the utility and validity of results generated from sorting tasks and was able to identify that sorting tasks produce results similar to that of conventional profiling methods. For example, sorting tasks performed without a list of descriptive terms produced similar results to the profile of beers. Sorting tasks were also concluded to be a robust tool but there are limits to the number of samples that can be evaluated without fatigue. Short term memory, assessor fatigue, and product complexity should be taken into consideration when planning sorting tasks to ensure maximum efficiency of sorting tasks. They also discovered that untrained assessors performed just as well as trained assessors, though the latter generated more groups. A study performed by Lelièvre et al. (2009) similarly found no effect of training on beer sorting, though they speculate that though assessors were trained, they were not beer experts and there are different standards in sensory expertise for the two groups. In a study performed by Brand et al (2018), the researchers aim to develop sorting methodologies that can be used by small-scale producers in-house to aid in quality assessment. The study combines sorting tasks with quality scores to develop and validate a method that is simple to perform can generate fast and scientific sensory data that can be related to quality. The researchers aim to increase access to quantitative sensory results that can be tailored to and easily accessible to industry professionals.

Currently, there appears to be only one study research that involves the application of sorting tasks with ciders. In the 2019 study, Jamir et al. compares the suitability of free sorting and flash profiling in characterization and comparison of cider products for small cider producers 
and determine differences between how American and Chinese consumers describe cider. This study is unique for the cider industry but there have been several studies that use sorting tasks in the wine and beer industry. The results of studies performed on wine and beer clearly illustrate the effectiveness of sorting studies as a research tool. The cost-effective and simplistic nature of sorting tasks allows for them to be used by untrained consumers and assessors, trained panels, and experts. The sorting task can be easily tailored depending on the research question and answer a wide range of questions that can aid in product development, quality assessment, and marketing.

\subsection{Conclusion}

Cider is an increasingly popular alcoholic beverage and regional and local ciders contribute greatly to its expansive growth. The majority of the currently available cider terms have been developed from research conducted at LARS in the 1970s and 1980s, which has not been updated since its publication. How ciders are perceived and described by consumers is relatively unknown. Though cider producers have expressed interest in the development of a consumerfocused descriptive lexicon, little research has been performed in this area. The literature showcases few scholarly resources on cider's sensory attributes and how consumers perceive, categorize, and describe them. In sensory research, there are few consumer-focused studies. The literature is scarcer for sensory research performed on ciders, craft or large distributors, in the US. Instead, sensory evaluation is used as a tool to assess sensory changes and attribute intensity or perception in ciders made under various conditions.

In cider research, sensory analysis is most often used as a tool to identify how processing, storage conditions, volatiles, cider composition, or any combination of the four may influence 
the sensory attributes of ciders. Few papers have a primary focus on profiling and characterizing the sensory attributes as perceived by cider consumers. Rather, trained panels of experienced assessors commonly evaluated research-produced cider's aroma and flavor in addition to indicating their liking for ciders produced under various conditions. Though the findings can be useful to cider makers in their development of best practices and quality control, the results cannot be immediately applied to consumer perceptions, liking, and categorization of ciders.

This study is split into two parts. First, a sorting task is used to profile the sensory attributes of Virginia ciders. Then, panelists evaluate the role of packaging and label on how they expect cider to taste and the occasion in which they would consume them. This can be useful in understanding where consumers are buying and drinking cider and as a first look into occasionbased consumption based on ciders' external product attributes. The findings will act as the foundation for future research on cider attributes and their role in consumers' willingness-to-pay, acceptance, and liking. It will also provide a glimpse into the descriptive terminology used by consumers, rather than professionals, and potentially act as the first step into a working cider lexicon that might be used by cider makers, professionals, and average and non-cider consumers. 


\section{References}

Abdi, H. (2007). RV and Congruence Coefficients. In Encyclopedia of Measurement and Statistics. https://doi.org/10.4135/9781412952644

Abdi, H., Dunlop, J. P., \& Williams, L. J. (2009). How to compute reliability estimates and display confidence and tolerance intervals for pattern classifiers using the Bootstrap and 3-way multidimensional scaling (DISTATIS). NeuroImage, 45(1), 89-95. https://doi.org/10.1016/j.neuroimage.2008.11.008

Alberti, A., Machado dos Santos, T. P., Ferreira Zielinski, A. A., Eleutério dos Santos, C. M., Braga, C. M., Demiate, I. M., \& Nogueira, A. (2016). Impact on chemical profile in apple juice and cider made from unripe, ripe and senescent dessert varieties. LWT - Food Science and Technology, 65, 436-443. https://doi.org/10.1016/j.lwt.2015.08.045

Anderson, H. (2002). Long Ashton Research Station: One hundred years of research. Pesticide Outlook, 13(5), 214-217. https://doi.org/10.1039/B209417G

Anton, M. J., Valles, B. S., Hevia, A. C., \& Lobo, A. P. (2014). Aromatic Profile of Ciders by Chemical Quantitative, Gas Chromatography-Olfactometry, and Sensory Analysis. Journal of Food Science, 79(1), S92-S99. https://doi.org/10.1111/1750-3841.12323

Aquilani, B., Laureti, T., Poponi, S., \& Secondi, L. (2015). Beer choice and consumption determinants when craft beers are tasted: An exploratory study of consumer preferences. Food Quality and Preference, 41, 214-224. https://doi.org/10.1016/j.foodqual.2014.12.005

Benivia, LLC. (2019). 2019 US Apple Crop Facts. Retrieved October 28, 2019, from https://www.pickyourown.org/USapplecrop.htm 
Bernot, K. (2017, February 13). Five cider statistic takeaways from CiderCon 2017. Retrieved October 28, 2019, from DRAFT website: https://draftmag.com/cider-statistics-20162017-cidercon/

Boudreau, T. F., Peck, G. M., O’Keefe, S. F., \& Stewart, A. C. (2017). The interactive effect of fungicide residues and yeast assimilable nitrogen on fermentation kinetics and hydrogen sulfide production during cider fermentation. Journal of the Science of Food and Agriculture, 97(2), 693-704. https://doi.org/10.1002/jsfa.8096

Brager, D. (2019). The Cider Rebound. Chicago, IL: Nielsen Holdings.

Brand, J., Kidd, M., van Antwerpen, L., Valentin, D., Næs, T., \& Nieuwoudt, H. H. (2018). Sorting in combination with quality scoring: A tool for industry professionals to identify drivers of wine quality rapidly. South African Journal of Enology and Viticulture, 39(2), 163-175. https://doi.org/10.21548/39-2-3203

Bruce, A., \& Bruce, P. (2017). Practical Statistics for Data Scientists. Retrieved from https://learning.oreilly.com/library/view/practical-statisticsfor/9781491952955/ch02.html\#Chapter_2

Chollet, S., Lelièvre, M., Abdi, H., \& Valentin, D. (2011). Sort and beer: Everything you wanted to know about the sorting task but did not dare to ask. Food Quality and Preference, 22(6), 507-520. https://doi.org/10.1016/j.foodqual.2011.02.004

Coton, E., \& Coton, M. (2016). Cider (Cyder; Hard Cider): The Product and its Manufacture. In Encyclopedia of Food and Health (pp. 119-127).

Crompton, M., \& Brager, D. (2017). U.S. Cider Trends: Increasing Your Odds of Sucess by Evaluating Marketplace Dynamics. 
Cyder Market. (2019). Cider Maker Survey. Retrieved October 28, 2019, from The Cyder Market website: http://cidermarket.com/cider-maker-survey.html

Danner, L., Ristic, R., Johnson, T. E., Meiselman, H. L., Hoek, A. C., Jeffery, D. W., \& Bastian, S. E. P. (2016). Context and wine quality effects on consumers' mood, emotions, liking and willingness to pay for Australian Shiraz wines. Food Research International, 89, 254-265. https://doi.org/10.1016/j.foodres.2016.08.006

Fabien-Ouellet, N., \& Conner, D. S. (2018). The Identity Crisis of Hard Cider. Journal of Food Research, 7(2), 54. https://doi.org/10.5539/jfr.v7n2p54

Garai-Ibabe, G., Ibarburu, I., Berregi, I., Claisse, O., Lonvaud-Funel, A., Irastorza, A., \& Dueñas, M. T. (2008). Glycerol metabolism and bitterness producing lactic acid bacteria in cidermaking. International Journal of Food Microbiology, 121(3), 253-261. https://doi.org/10.1016/j.ijfoodmicro.2007.11.004

Hawkins, J. (2018). Textual Analysis. In M. Allen (Ed.), The SAGE Encyclopedia of Communication Research Methods (pp. 1754-1756). https://doi.org/10.4135/9781483381411.n623

Hopfer, H., \& Heymann, H. (2014). Judging wine quality: Do we need experts, consumers or trained panelists? Food Quality and Preference, 32, 221-233. https://doi.org/10.1016/j.foodqual.2013.10.004

Hout, M. C., Papesh, M. H., \& Goldinger, S. D. (2013). Multidimensional scaling. Wiley Interdisciplinary Reviews. Cognitive Science, 4(1), 93-103. https://doi.org/10.1002/wcs.1203

Lachowicz, S., Oszmiański, J., Uździcka, M., Chmielewska, J., Escuer, M. C., \& CampsBossacoma, M. (2019). The Influence of Yeast Strain, $\beta$-Cyclodextrin, and Storage Time 
on Concentrations of Phytochemical Components, Sensory Attributes, and Antioxidative Activity of Novel Red Apple Ciders. Molecules, 24(13), 2477.

https://doi.org/10.3390/molecules24132477

Lahne, J., Collins, T. S., \& Heymann, H. (2016). Replication Improves Sorting-Task Results Analyzed by DISTATIS in a Consumer Study of American Bourbon and Rye Whiskeys. Journal of Food Science, 81(5), S1263-S1271. https://doi.org/10.1111/1750-3841.13301

Lawless, H. T., \& Heymann, H. (2010). Sensory Evaluation of Food: Principles and Practices (2nd ed). New York: Springer.

Lea, A. G. H., \& Arnold, G. M. (1978). The phenolics of ciders: Bitterness and astringency. Journal of the Science of Food and Agriculture, 29(5), 478-483. https://doi.org/10.1002/jsfa.2740290512

Lea, A. G. H., \& Drilleau, J. (2003). Cidermaking. In A. G. H. Lea \& J. R. Piggott (Eds.), Fermented Beverage Production (pp. 59-87). https://doi.org/10.1007/978-1-4615-01879_4

Lelièvre, M., Chollet, S., Abdi, H., \& Valentin, D. (2008). What is the validity of the sorting task for describing beers? A study using trained and untrained assessors. Food Quality and Preference, 19(8), 697-703. https://doi.org/10.1016/j.foodqual.2008.05.001

Lelièvre, M., Chollet, S., Abdi, H., \& Valentin, D. (2009). Beer-Trained and Untrained Assessors Rely More on Vision than on Taste When They Categorize Beers. Chemosensory Perception, 2(3), 143-153. https://doi.org/10.1007/s12078-009-9050-8

Lockshin, L., \& Corsi, A. M. (2012). Consumer behaviour for wine 2.0: A review since 2003 and future directions. Wine Economics and Policy, 1(1), 2-23. https://doi.org/10.1016/j.wep.2012.11.003 
Ma, S., Neilson, A., Lahne, J., Peck, G., O’Keefe, S., Hurley, E. K., ... Stewart, A. (2018). Juice Clarification with Pectinase Reduces Yeast Assimilable Nitrogen in Apple Juice without Affecting the Polyphenol Composition in Cider. Journal of Food Science, 83(11), 27722781. https://doi.org/10.1111/1750-3841.14367

Ma, S., Neilson, A. P., Lahne, J., Peck, G. M., O’Keefe, S. F., \& Stewart, A. C. (2018). Free amino acid composition of apple juices with potential for cider making as determined by UPLC-PDA. Journal of the Institute of Brewing, 124(4), 467-476. https://doi.org/10.1002/jib.519

Malone-Brown, E. (2014, July 17). Cider History. Retrieved October 16, 2019, from A Very Brief History of Cider website: https://web.archive.org/web/20140717030425/https://cydermarket.com/History_of_Cider .html

Meilgaard, M. C., Dalgliesh, C. E., \& Clapperton, J. F. (1979). Beer Flavour Terminology. Journal of the Institute of Brewing, 85(1), 38-42. https://doi.org/10.1002/j.20500416.1979.tb06826.x

Melcher, J. M., \& Schooler, J. W. (1996). The Misremembrance of Wines Past: Verbal and Perceptual Expertise Differentially Mediate Verbal Overshadowing of Taste Memory. Journal of Memory and Language, 35(2), 231-245. https://doi.org/10.1006/jmla.1996.0013

Mora, M., Giussani, B., Pagliarini, E., \& Chaya, C. (2019). Improvement of an emotional lexicon for the evaluation of beers. Food Quality and Preference, 71, 158-162. https://doi.org/10.1016/j.foodqual.2018.06.007 
NCCS. (n.d.). Hierarchical Clustering / Dendrograms. Retrieved from https://ncsswpengine.netdna-ssl.com/wpcontent/themes/ncss/pdf/Procedures/NCSS/Hierarchical_Clustering-Dendrograms.pdf New York Cider Association. (2019). New York Cider Association. Retrieved November 17, 2019, from New York Cider Association website: https://www.newyorkciderassociation.com/about

Nielsen. (2018). United States Association of Cider Makers and Nielsen Expand Their Analytic Relationship. Retrieved October 16, 2019, from https://www.nielsen.com/us/en/pressreleases/2018/united-states-association-of-cider-makers-and-nielsen-expand-relationsip

Nilsson, I., Reid, N., \& Lehnert, M. (2018). Geographic Patterns of Craft Breweries at the Intraurban Scale. The Professional Geographer, 70(1), 114-125. https://doi.org/10.1080/00330124.2017.1338590

Noble, A. C., Arnold, R. A., Buechsenstein, J., Leach, E. J., \& Schmidt, J. O. (1987). Modification of a Standardized System of Wine Aroma Terminology. 38(2), 4.

Nurin, T. (2018). Hard Cider Sales Slip And Then Rebound. Retrieved October 16, 2019, from Forbes website: https://www.forbes.com/sites/taranurin/2018/02/28/hard-cider-sales-slipthen-rebound/

Peng, B., Li, F., Cui, L., \& Guo, Y. (2015). Effects of Fermentation Temperature on Key Aroma Compounds and Sensory Properties of Apple Wine. Journal of Food Science, 80(12), S2937-S2943. https://doi.org/10.1111/1750-3841.13111

Peng, B., Yue, T., \& Yuan, Y. (2009). Analysis of key aroma components in cider from Shaanxi (China) Fuji apple. International Journal of Food Science \& Technology, 44(3), 610615. https://doi.org/10.1111/j.1365-2621.2008.01875.x 
Qin, Z., Petersen, M. A., \& Bredie, W. L. P. (2018). Flavor profiling of apple ciders from the UK and Scandinavian region. Food Research International, 105, 713-723. https://doi.org/10.1016/j.foodres.2017.12.003

Raz, C., Piper, D., Haller, R., Nicod, H., Dusart, N., \& Giboreau, A. (2008). From sensory marketing to sensory design: How to drive formulation using consumers' input? Food Quality and Preference, 19(8), 719-726. https://doi.org/10.1016/j.foodqual.2008.04.003

Rivaroli, S., Baldi, B., \& Spadoni, R. (2020). Consumers' perception of food product craftsmanship: A review of evidence. Food Quality and Preference, 79, 103796. https://doi.org/10.1016/j.foodqual.2019.103796

Rorabaugh, W. J. (1976). Estimated U.S. Alcoholic Beverage Consumption, 1790-1860. Journal of Studies on Alcohol, 37(3), 8.

Rosend, J., Kuldjärv, R., Rosenvald, S., \& Paalme, T. (2019). The effects of apple variety, ripening stage, and yeast strain on the volatile composition of apple cider. Heliyon, 5(6), e01953. https://doi.org/10.1016/j.heliyon.2019.e01953

Rupp, becca. (2015). The Highs and Lows of Hard Apple Cider History. Retrieved November 27, 2019, from National Geographic website: https://www.nationalgeographic.com/culture/food/the-plate/2015/10/08/the-highs-andlows-of-hard-apple-cider-history/

Sánchez, A., de Revel, G., Antalick, G., Herrero, Mó., García, L. A., \& Díaz, M. (2014). Influence of controlled inoculation of malolactic fermentation on the sensory properties of industrial cider. Journal of Industrial Microbiology \& Biotechnology. 
Scott, J. A., \& Swaffield, C. H. (1998). Observations on the Influence of Temperature, Dissolved Oxygen and Juice Source on Stored Alcoholic Cider Flavour Development. Food Biotechnology, 12(1-2). https://doi.org/10.1080/08905439809549940

Spence, C., Wan, X., Woods, A., Velasco, C., Deng, J., Youssef, J., \& Deroy, O. (2015). On tasty colours and colourful tastes? Assessing, explaining, and utilizing crossmodal correspondences between colours and basic tastes. Flavour, 4(1), 23. https://doi.org/10.1186/s13411-015-0033-1

Suwonsichon, S. (2019). The Importance of Sensory Lexicons for Research and Development of Food Products. Foods, 8(1). https://doi.org/10.3390/foods8010027

Symoneaux, R., Chollet, S., Patron, C., Bauduin, R., Le Quéré, J. M., \& Baron, A. (2015). Prediction of sensory characteristics of cider according to their biochemical composition: Use of a central composite design and external validation by cider professionals. $L W T$ Food Science and Technology, 61(1), 63-69. https://doi.org/10.1016/j.lwt.2014.11.030

Symoneaux, R., Guichard, H., Le Quéré, J. M., Baron, A., \& Chollet, S. (2015). Could cider aroma modify cider mouthfeel properties? Food Quality and Preference, 45, 11-17. https://doi.org/10.1016/j.foodqual.2015.04.004

ten Kleij, F., \& Musters, P. A. D. (2003). Text analysis of open-ended survey responses: A complementary method to preference mapping. Food Quality and Preference, 14(1), 4352. https://doi.org/10.1016/S0950-3293(02)00011-3

The Cider Journal. (2017). Artisan Cider Sales Surge in 2016. Retrieved November 10, 2019, from The Cider Journal website: https://ciderjournal.com/artisan-cider-sales-surge-2016/ The Virginia Apple Board. (2019). Virginia Apples. Retrieved November 16, 2019, from Virginia Apple Board website: https://www.virginiaapples.net 
Thériault, F. (2019, February 20). Sector Trend Analysis - Cider market trends in the United States and the United Kingdom [Organizational description]. Retrieved October 16, 2019, from http://www.agr.gc.ca/eng/industry-markets-and-trade/international-agri-foodmarket-intelligence/reports/sector-trend-analysis-cider-market-trends-in-the-unitedstates-and-the-united-kingdom/?id=1549381895074

Thompson-Witrick, K. A., Goodrich, K. M., Neilson, A. P., Hurley, E. K., Peck, G. M., \& Stewart, A. C. (2014). Characterization of the Polyphenol Composition of 20 Cultivars of Cider, Processing, and Dessert Apples (Malus $\times$ domestica Borkh.) Grown in Virginia. Journal of Agricultural and Food Chemistry, 62(41), 10181-10191. https://doi.org/10.1021/jf503379t

Tozer, P. R., Galinato, S. P., Ross, C. F., Miles, C. A., \& McCluskey, J. J. (2015). Sensory Analysis and Willingness to Pay for Craft Cider. Journal of Wine Economics, 10(3), 314328. https://doi.org/10.1017/jwe.2015.30

USACM. (2019). Cider Lexicon Project. Retrieved November 1, 2019, from https://ciderassociation.org/cider-lexicon-project/

Varela, P., \& Ares, G. (Eds.). (2014). Sorting Tasks. In Novel Techniques in Sensory Characterization and Consumer Profiling. https://doi.org/10.1201/b16853

Williams, A. A. (1974). Flavour Research and the Cider Industry. Journal of the Institute of Brewing, 80(5), 455-470. https://doi.org/10.1002/j.2050-0416.1974.tb06795.x

Williams, A. A., Lea, A. G. H., \& Timberlake, C. F. (1977). Measurement of Flavor Quality in Apples, Apple Juices, and Fermented Ciders. In R. A. Scanlan (Ed.), Flavor Quality: Objective Measurement (Vol. 51, pp. 71-88). https://doi.org/10.1021/bk-19770051.ch006 
Williams, Anthony A. (1975). The development of a vocabulary and profile assessment method for evaluating the flavour contribution of cider and perry aroma constituents. Journal of the Science of Food and Agriculture, 26(5), 567-582.

https://doi.org/10.1002/jsfa.2740260503

WSU Extension. (2019). History of Cider. Retrieved October 16, 2019, from WSU Cider website: https://cider.wsu.edu/history-of-cider/

Ye, M., Yue, T., \& Yuan, Y. (2014). Changes in the profile of volatile compounds and amino acids during cider fermentation using dessert variety of apples. European Food Research and Technology, 239(1), 67-77. https://doi.org/10.1007/s00217-014-2204-1 


\section{Chapter 3: Experimental}

\subsection{Introduction}

In the United States (US), the term "hard cider" refers to an alcoholic beverage made from fermented apple juice and "cider" by itself refers to unpasteurized and unfiltered apple juice. In the rest of the world, however, "cider" means the alcoholic, fermented beverage produced from apple juice (Lea, 1995). In this paper, for brevity and consistency, the term "cider" will be used for the alcoholic beverage.

In the past ten years alone, the US cider industry has grown exponentially. In 2008, cider only made up $0.06 \%$ of the alcoholic beverage industry, with sales totaling $\$ 44$ million in 2008 (Nielsen, 2018; Nurin, 2018). In 2018, cider made up $0.4 \%$ of the alcoholic beverage industry and sales reached $\$ 506$ million (Nielsen, 2018), (Nurin, 2018). US cider sales are expected to hit $\$ 1.67$ billion by 2022 (Thériault, 2019). In 2018, cider growth, as a whole, was faster than that of craft beer and the alcoholic beverage market in total, with regional and local brands as large contributors.

These trends are indicative of a rapidly expanding industry with small- and medium-sized producers contributing to its expansion, however, cider makes up less than $1 \%$ of the alcoholic beverage market share. There is little research on differentiating cider styles and the demand for

cider made with specific apple cultivars (Fabien-Ouellet \& Conner, 2018). There also appears to be a gap in knowledge in what the general public knows and understands of cider. In the US, non-cider drinkers do not necessarily know what cider is, where it comes from, or how it is made. There are opportunities to grow the cider industry through education, marketing, and advertising (Fabien-Ouellet \& Conner, 2018; Thériault, 2019). 
Much of the early research conducted on ciders can be traced back to the Long Ashton Research Station (LARS). During the 1970s and 1980s, research conducted on cider included, but was not limited to, working to define the current framework of flavor research and the cider industry (A. A. Williams, 1974), formulating sensory, physical, and analytical methods to assess the quality of apples, apple juice, and cider (Andrew G. H. Lea \& Arnold, 1978; A. A. Williams, Lea, \& Timberlake, 1977), and developing a descriptive vocabulary and profiling method for cider and perry aroma constituents through gas chromatography (GC) and sensory analysis (A.A. Williams, 1975). This research provides much of the current framework of cider flavor chemistry, but a large body of work at LARS was concluded or left unfinished upon its closure in 1981 (Anderson, 2002). A 2019 literature indicated that there is little evidence that the descriptive cider vocabulary developed by Williams has been built upon since its original publication in 1975 .

In current cider research, sensory analysis is most often used as a tool to identify how processing, storage conditions, volatiles, cider composition, or any combination of the four may influence the sensory attributes of ciders. Few papers have a primary focus on profiling and characterizing the sensory attributes. Rather, trained panels of experienced assessors commonly evaluated research-produced cider's aroma and flavor in addition to indicating their liking for ciders produced under various conditions. Published cider research that incorporate sensory analysis are those that aimed to understand the contribution of cider composition, flavor and aroma compounds and perceived sensory attributes (Anton, Valles, Hevia, \& Lobo, 2014; Peng, Yue, \& Yuan, 2009; Symoneaux, Guichard, Le Quéré, Baron, \& Chollet, 2015); assessed how processing and storage parameters impact the sensory attributes of cider (Lachowicz et al., 2019; Peng, Li, Cui, \& Guo, 2015; Sánchez et al., 2014; Scott \& Swaffield, 1998); and generated a 
predictive model evaluating the impact of apple procyanidins, fructose, acid content, and ethanol on astringency, sweetness, and sourness of ciders (Symoneaux, Chollet, et al., 2015). These studies, with the exception of Symoneaux et al. (2015), also incorporated trained and/or experienced sensory panels to assess and describe the sensory attributes of ciders produced under varying conditions. Symoneaux et al. (2015) used two separate panels, one consisting of trained sensory assessors and another consisting of expert cider professionals, to validate a predictive model that aimed to use measurements and sensory contributions of malic acid, fructose, ethanol, and type and quantity of procyanidins to predict the sensory attributes of ciders based on their composition.

These studies build on the work conducted at LARS in the 1970s and 1980s provide increased insight on the correlation of cider's chemistry processing and storage parameters on its resulting sensory attributes. This work can be used to guide the development of best practices in cider making, but it is unclear how these ciders might be perceived by consumers. Specifically, there are opportunities to expand on this research through sensory analysis of ciders with consumers of varying cider knowledge to further understand how they may perceive, categorize, or describe ciders.

The current language used to describe ciders has primarily been developed by and used among cider experts who may or may not be in communication with each other, unconsciously generating discrepancies in cider styles and descriptive language. Professional organizations like the United States Cider Maker Association (USACM) and the Great Lakes International Cider Perry Competition (GLINTCAP) have developed cider style guides and descriptive vocabulary to be used by cider makers and professionals (GLINTCAP, 2019; USACM, 2018).

CIDERCRAFT magazine worked alongside cider experts to develop a tasting guide with 
categories and a flavor wheel. Oftentimes, how ciders are categorized and described is organization-dependent, creating resources that may categorize or describe the same cider styles in varying ways.

Another potential issue with professional and expert-generated terminology is that many studies have identified significant differences between the terminology used by trained assessors, product experts, and novice tasters (Hopfer \& Heymann, 2014; Lelièvre, Chollet, Abdi, \& Valentin, 2008; Melcher \& Schooler, 1996). Thus, there are opportunities to define cider styles and categories with a descriptive sensory language, or lexicon, that can be used universally by both non-cider drinkers and cider experts during selection, consumption, and discussion. Further research in this area should include collaboration with cider experts and average and non-cider consumers to develop and apply universal cider. Additionally, more work in this area is necessary to develop resources that have the potential to improve how cider is discussed and described by both the average consumer and cider experts.

Cider is a rapidly expanding industry and as consumer interest increases, it is best to match consumer expectation of extrinsic product attributes with experienced sensory attributes to ensure repurchasing through customer satisfaction, retention, and brand loyalty. Repeat purchasing is a necessity for a producer's success, especially that of small- and medium-sized cider producers. Drivers of repeat purchasing can include expectations generated from both extrinsic product attributes (e.g., packaging type, brand, price, and label) and actual sensory experience during consumption (Hamza, 2014; Mueller, Osidacz, Francis, \& Lockshin, 2010).

The current paper seeks to address this gap in knowledge and begin identifying how cider is perceived, described, and categorized cider, using sorting tasks, a rapid sensory analysis method. Free sorting tasks involve panelists evaluating and sorting all products in one session. 
Panelists are asked to first look, smell, and/or taste products and then sort them into mutually exclusive groups based on their perceived product similarities. They are allowed to then describe their groupings using any criteria they like. Sorting tasks are used in sensory analysis to collect similarity data "in which each assessor groups together stimuli based on their perceived similarities" (Varela \& Ares, 2014, p. 207). Statistical analysis of sorting data can reveal the structures of the product space and interpret its underlying dimensions (categories) (Varela \& Ares, 2014). Pairing the analysis of sorting data with textual analysis of descriptors used by panelists allows for further extrapolation of not only how panelists group products but what attributes influence these patterns.

There are two parts to this study: In study 1, panelists smelled and tasted cider samples and asked to evaluate their aroma and flavor. In study 2, panelists were asked to visually evaluate photos of the cider samples' packaging and label. Chemical analysis of cider was also performed to gain a preliminary profile of cider chemistry and descriptors used by panelists. The goals of this study are 1) to begin defining how cider is described and categorized using rapid sensory methodology; 2) to explore the influence of product packaging attributes on how panelists expect a cider to taste and the occasions they would most likely consume them in; and 3) to identify if panelists expectations match what is actually experienced. 


\subsection{Materials and Methods}

\subsubsection{Study 1: Free Sorting of Ciders Through Aroma and Flavor Analysis}

\subsubsection{Samples}

In Summer 2018, the Cider Guide's Virginia Cider Map and Directory (West, 2019) was used to identify 18 cideries that were currently producing cider in Virginia (Table 1). These cideries were contacted by phone or e-mail and asked to identify one cider that they believed was representative of their cidery, style, and/or cider making philosophy. Of those 18 , eleven cideries responded directly to this request and named one cider that represented their establishment, which was then included in this sorting study. For the remaining seven cideries, the top-ranked "traditional" cider identified by The Cyder Market website (https://www.cydermarket.com/) was identified and included in the sorting study.

One cider was donated by the producer and the remaining ciders were purchased from both local producers and retailers and stored in their original packaging at room temperature prior to sensory analysis. One cider was stored refrigerated at 38 degrees $\mathrm{F}$ due to evident fermentation activity in the bottle. On test day, this cider was allowed to reach room temperature overnight before being poured and sampled.

Each of the 18 ciders was presented as a $20 \mathrm{ml}$ aliquot in an opaque, 15-ounce black wine glass to conceal obvious visual differences, such as color or carbonation, which might influence panelist perceptions during sensory analysis. A watch glass was placed on top of each glass to limit the loss of volatile compounds from the sample. All samples were labeled with random 3digit codes and presented to panelists at room temperature. Samples were poured 30 minutes prior to analysis and discarded 1 hour after pouring. All ciders were left at room temperature after preparation and covered with a stopper or parafilm to minimize carbonation loss before 
being served to panelists. Halfway through the six-hour study, all remaining cider samples in open packaging were discarded and replenished with a new bottle or can to limit the effects of carbonation loss on the sensory characteristics of the products.

\subsubsection{Panelists}

Panelists $\left(N_{1}=65\right)$ were recruited from a large college campus in the United States and the surrounding community through university e-mail listservs, word-of-mouth, and via social media networks. Potential panelists were required to complete a brief screening survey to determine their age, eligibility, and consumption of alcoholic beverages. Panelists who completed and passed the screening survey (Appendix 1) then scheduled a 30-minute time slot to participate in sensory analysis of samples.

All recruitment and scheduling was managed using Compusense Cloud sensory management software (Guelph, ON). All panelists were untrained, at least 21 years of age, had no allergies to alcohol, apples, or pears, and had consumed alcoholic beverages on at least one previous occasion. On day one of sensory analysis, panelists $\left(N_{1}=16\right)$ were presented with 17 of the 18 selected ciders after only receiving enough of the donated cider for two days. On days two and three, panelists $\left(N_{1}=49\right)$ evaluated all 18 ciders, including the cider excluded from day one. This study was approved by the Virginia Tech Institutional Review Board, protocol 171132. 


\subsubsection{Sorting Task Procedure}

After logging into Compusense, panelists $\left(N_{1}=65\right)$ were presented concurrently with $\left(K_{1}=17\right.$ or 18$)$ ciders. They were instructed to evaluate the aroma and flavor of the ciders and then sort them into mutually exclusive groups based on their perception of product similarities (Appendix 2). They were free to sort ciders, based on any criteria they chose, into at least 2 groups and no more than $K_{1}-1$ groups. They were then asked to describe each group using their own words.

Panelists were instructed to expectorate the samples after analysis and cleanse their palate with room temperature water and a bite of unsalted saltine crackers. Following the completion of this task, panelists were asked to complete a brief demographic questionnaire and a survey about their cider and other alcohol consumption and purchasing habits (Appendix 2).

\subsubsection{Data Analysis}

Data were analyzed using DISTATIS (through the DistatisR package (Beaton, et. al, 2019)). DISTATIS is an extension of multidimensional scaling (MDS) that accounts for differences of individual assessors prior to the generation of the consensus product space by calculating the $R_{\mathrm{V}}$ coefficient, a measure of similarity between aggregated assessor sorting matrices (Abdi et. al, 2007). The result is a 2-dimensional map that conveys, spatially, the relationships among items, wherein similar products are located closer to one another, and dissimilar products are located further apart (Hout et al., 2013). Visual 95\% bootstrap confidence ellipses were projected onto the map for statistical inferences of product differences and similarities. The method, its specific calculations, and applications have been described in detail in the literature ( Abdi et al., 2007; Abdi, Valentin, \& Ub, 2007; Abdi et al.,2009; Lahne et al., 
2016; Lahne et al., 2018). Hierarchical cluster analysis (HCA) was performed using Ward's method on first and second dimensions of the cross-product agreement matrix to identify clusters of ciders that are most similar (through the function hclust in the stats package (R Core Team, 2019)). Panelist-generated group descriptors were analyzed through classical text analysis where descriptors were lemmatized and de-stemmed (through the R packages tidytext (De Queiroz et al., 2019) and textstem (Rinker, 2018)). Two individual researchers then analyzed text separately to synonymize terms into categories and then discussed results to form a consensus. Descriptors that were used by at least 4 panelists were then projected onto the DISTATIS product map to illustrate common sensory terms applied to ciders.

\subsubsection{Free Sorting of Ciders Through Visual Evaluation of Cider Packaging and Labels}

\subsubsection{Samples}

The 18 ciders evaluated for aroma and flavor (Section 2.1) were also evaluated in a second study to examine how panelists categorize and describe ciders based solely on their packaging and labels. Due to the large number of samples, photos of ciders were taken and presented to the panelists for ease of handling, instead of actual bottles and cans $\left(K_{2}=18\right)$. Cider photos were labeled with 3-digit codes that were independent and different from those in the flavor and aroma study (Section 2.1).

Photos were taken with a LimoStudio Table Top lightbox, two LimoStudio 5500K 600 Lumen LED lights, Nikon D3100 camera, and an 18-oz red plastic cup for size reference.

Each cider was represented by a set of four photos. Two close-up photos (front and back of the packaging) were taken to showcase any verbiage, labels, photos, and other defining attributes of the packaging (Figure 1). Two photos (front and back of the packaging) were then 
taken of the full package next to a red plastic cup to demonstrate the size of the product and create a reference in comparison to other samples (Figure 2). These photos were printed in color on 4-inch x 6-inch glossy photo paper and organized into photo sheets, with one sheet holding the four photos representing each sample. Each photo sheet was assigned 3-digit randomized codes for the samples, which were independent of those in the first flavor and aroma study (Section 2.1).

\subsubsection{Panelists}

After participating in the aroma and flavor sorting study of ciders, panelists were invited but not required to participate in the visual evaluation sorting study. Panelists $\left(N_{2}=63\right)$ were untrained, at least 21 years of age, and had consumed alcoholic beverages on at least one previous occasion. This part of the study was also approved by the Virginia Tech Institutional Review Board, protocol 17-1132.

\subsubsection{Sorting Task Procedure}

Panelists $\left(N_{2}=63\right)$ were presented with a binder containing all cider sample photo sheets $\left(K_{2}=18\right)$ from which they were able to remove the pages for the sorting tasks. They were asked to sort the photo sheets according to two prompts: 1) how they thought the ciders would taste and 2) what occasion they would be most likely to drink them at. To avoid order effects, panelists were randomized into one of two tests that either displayed the "taste" or “occasion” sorting task prompt first. For each sorting prompt, they were free to sort ciders, based on any criteria they chose, into at least two groups and no more than $K_{2}-1$ groups. Paper-clips 
were provided to assist panelists in organizing the photo sheet groups (Figure 3). They were then asked to label each group using their own words (Appendices 3 and 4).

\subsubsection{Data Analysis}

The data generated from study 2 were analyzed separately using the same procedures outlined in section 2.1.4.

\subsubsection{Chemical Analysis}

A 150-ml aliquot of each sample was collected for chemical analysis at the end of the study. Each sample was flushed with nitrogen and degassed prior to quantification of the following parameters: $\mathrm{pH}$ (Accumet Ultra Triode Electrode Model 13-620-631, Thermo Fisher Scientific, Waltham, MA, USA); titratable acidity (standard method as reported previously by others (Ough \& Amerine, 1988)); and residual sugars (standard method as previously reported by others with some modifications (Yu, Yuan, Fu, \& Zhu, 2016)). Samples were filtered and $1 \mathrm{ml}$ of the supernatant was transferred into a 2-ml glass high-performance liquid chromatography (HPLC) vessel with $1 \mathrm{ml}$ of acetonitrile. Residual sugar identification and quantification was performed with Luna Omega $3 \mu$ m SUGAR $100 \AA$ A , LC Column $(250$ x 4.6 mm) with a column temperature of 40 degrees C. A 1-ml aliquot of each sample was injected, and sugars were eluted with $75 \%$ acetonitrile and $25 \% \mathrm{HPLC} \mathrm{H}_{2} \mathrm{O}$ at a flow rate of $1.0 \mathrm{ml} / \mathrm{min}$. One-way analysis (ANOVA) was performed to identify differences among parameters and if significant differences were identified. Tukey's HSD was used as a post hoc test to perform pairwise comparisons between ciders. 


\subsection{Results and Discussion}

\subsubsection{Demographic Survey and Questionnaire}

The majority of the panelists were female and their ages ranged from 21 to 70 years. Most purchased cider once a month or once a week and consumed it between less than once a month and 1-4 times a month. Regarding interest in alcoholic beverages other than cider, panelists primarily reported wine or beer consumption (Table 2). Panelists were asked: "When purchasing cider, what is most [important, appealing, or influential] to you?" Of those who responded $\left(N_{1}=60\right)$, the most influential factor in driving cider purchase or consumption was anticipated or previously experienced sensory attributes (e.g., "sweet", "fruity") followed by cost, location produced, fruit variety (e.g., “apple type”, “pineapples”), newly or previously tasted, brand, and label (Table 3). This information suggests the importance of cider producers to adequately set and meet their ciders' sensory attributes.

\subsubsection{Chemical analysis}

One-way analysis of variance (ANOVA) identified significant differences among all chemical parameters (Table 4). Tukey's HSD identified groupings of cider based on ciders' chemical parameters. The groupings were not predictive in how they were sorted, indicating a more complex relationship between cider chemistry and experienced sensory attributes.

The $\mathrm{pH}$ of ciders ranged between 3.40 and 3.95. Producer-reported alcohol content (ethanol) ranged from 4.7 to $8.6 \%$. Titratable acidity (measured as malic acid equivalents) ranged from 0.94 to $3.40 \mathrm{~g} / \mathrm{L}$. Total residual sugars ranged from 1.38 to $35.67 \mathrm{~g} / \mathrm{L}$. Separately measured individual sugars, fructose, and glucose, ranged from 0.00 to $14.36 \mathrm{~g} / \mathrm{L}$ and $1.38-23.55 \mathrm{~g} / \mathrm{L}$, respectively. 


\subsubsection{Study 1: Flavor and Aroma Analysis of Ciders}

Eighteen ciders were sorted into groups and those groups were given textual descriptors by 49 panelists. Data from the remaining 16 panelists were omitted due to the absence of cider 12 from the dataset during the first day of the study. The data from these 16 panelists were analyzed separately and did not differ markedly from the main analysis of 49 panelists who evaluated the full sample set (Appendix Figure 1). Panelists made at least 2 groups and at most 14 groups. Panelists collectively used 341 unique descriptors to label their groupings which were reduced through textual analysis to 37 terms. "Cider" is abbreviated to "C" when discussing specific examples from this study.

Subjects agreed substantially on sorting configurations for the ciders with the first principal component of the subject agreement matrix explaining most of the variation $(\lambda=0.249, \tau=25 \%)$ and a considerable drop in the second dimension $(\lambda=0.0438, \tau=4 \%)$. In the resulting consensus plot (Figure 4), the first dimension separated ciders which panelists labeled as tart, dry, bitter, or characterless from those labeled as herbal, sweet, tangy, floral, and fruity. The second principal component separated ciders labeled as characterless, funky, light, bitter, or wine-like from those labeled as crisp, floral, tart, carbonated, or apple or pear-like. Hierarchical clustering of dimensions 1 and 2 of the cross-product agreement matrix revealed 3 major clusters of ciders: cluster 1 (colored blue in Figure 4) primarily consists of tart ciders, cluster 2 (colored purple in Figure 4) primarily consists of dry and bitter ciders, and cluster 3 (colored green in Figure 4) primarily consists of sweet and fruity ciders. 


\subsubsection{Study 2: Visual Evaluation of Cider Packaging and Labels}

Eighteen cider photo sheets were sorted by 63 panelists into groups through visual evaluation of the ciders' packaging and labels. Based on how panelists expected a cider to taste, panelists made at least 2 groups and at most 8 groups and collectively used 180 unique descriptors to label their groupings, which were reduced through textual analysis to 28 terms. Based on the occasion panelists might consume a cider in, panelists made at least 2 groups and at most 8 groups and collectively used 209 unique descriptors to label their groupings, which were reduced through textual analysis to 28 terms. For clarity, the two conditions are discussed separately below. "Cider" is abbreviated to " $\mathrm{C}$ " when discussing specific examples from this study.

\subsubsection{Visual Evaluation: Panelists Expectations of Cider Taste}

Subjects agreed substantially on the expected taste of the ciders with the first principal component of the subject agreement matrix explaining most of the variation $(\lambda=0.231, \tau=$ $24 \%)$ and a considerable drop in the second dimension $(\lambda=0.0494, \tau=5 \%)$. In the resulting consensus plot (Figure 5), the first dimension separated ciders panelists expected to taste apple/pear-like, tart, sweet, or carbonated from those expected to be tart earthy, funky, decadent, dry, or complex. The second principal component separated ciders expected to taste bitter, earthy, barrel-aged, funky, or decadent from those expected to be sweet, carbonated, fruity, light, or crisp from. Hierarchical clustering of dimensions 1 and 2 of the cross-product agreement matrix revealed 4 distinct clusters of ciders: cluster 1 (colored purple in Figure 5) contains ciders thought to be tart, cluster 2 (colored green in Figure 5) contains ciders thought to be crisp, carbonated, or light, cluster 3 (colored blue in Figure 5) contained ciders thought to be fruity or 
light, and cluster 4 (colored pink in Figure 5) contains cider thought to be complex, unfiltered, alcoholic, or dry.

Cluster 1 in Figure 5 shows a tight cluster of ciders that panelists expected to be "tart". These ciders $(\mathrm{C} 4, \mathrm{C} 5, \mathrm{C} 12)$ are unique from others in this sample set as they share similar attributes of deep or bright green coloring on a 16-ounce metallic can (example found in figure 9). This pattern may be explained by the association of the color green with green apples, a very tart apple that panelists may be familiar with, in addition to the colors like yellow or green being commonly associated with sourness (Spence et al., 2015). However, C7 is a canned cider that was not grouped with those in cluster 1 . Instead, it can be found in cluster 2, alongside ciders that are described as "carbonated" or "full-bodied". These ciders (C2, C7, C9, C10) show many similarities to the packaging and labels of beer. This might be explained by the fact that many panelists stated that they also consume beer in addition to cider (Table 2). Though large in size at $750 \mathrm{ml}, \mathrm{C} 2$ is the only cider in clear packaging with a color that is similar to many commercially-available beers. The other ciders are smaller than others included in this study with dark brown bottles with metallic tops. Ciders in this group have similar color schemes of warm yellows, reds, oranges, and browns.

Cluster 3 contains ciders $(\mathrm{C} 1, \mathrm{C} 3, \mathrm{C} 8, \mathrm{C} 13, \mathrm{C} 16)$ thought to taste "fruity" and are all packaged in dark green glass and sealed with a metallic top and range from 500 to $750 \mathrm{ml}$ in size, putting them in a size category similar to commercially available wines. Most labels are similar in spacing and design with bright whites, greens, and blues in their design with most having a small image of fruit or animal in a similar location (example found in figure 10). Having light or less intense colored labels may also be associated with healthier foods and this, combined with the aforementioned product attributes, may explain why panelists expected these ciders to be 
"light" and "fruity" (Mai, Symmank, \& Seeberg-Elverfeldt, 2016). This may explain why C8, the only one with a nutrition label that displays low-calories and sugars, was found in this cluster even though it is bright red, and why $\mathrm{C} 4$, found in cluster 1, was excluded with its bright green and dark blue coloring, even with an explicit "low-cal cider, only 115 calories" claim on the bottom of the label.

Ciders in cluster 4 (C6, C11, C14, C15, C17, C18) were thought to taste "alcoholic", "complex", or "unfiltered"; these attributes are shared with that of wine. All ciders, with the exception of $\mathrm{C} 14$, were packaged into 750-ml glass bottles ranging from clear to dark brown. All labels had images and/or fonts that were large in size (example found in figure 11). C14 was the cider most associated with the term "flawed" and does not overlap with other ciders in this cluster, indicating that it may be a poor fit. This may be attributed to a cartoon drawing of a farmhouse and animal on the label, but further research should be performed to explore consumer perceptions.

\subsubsection{Visual Evaluation: Occasions Panelists Might be Inclined to Consume Ciders At}

Subjects agreed substantially on what occasions they may be inclined to consume the ciders at, with the first principal component of the subject agreement matrix explaining most of the variation $(\lambda=0.300, \tau=30 \%)$ and dropping significantly in the second $(\lambda=0.0602$, $\tau=6 \%$ ) dimension. In the consensus plot of the first and second principal component (Figure 6), the first principal component separated ciders panelists might consume at home, seasonal events during the summer or holidays, or with food or relaxation from ciders that might be consumed at tailgates, celebrations, or a bar. The second principal component separates ciders panelists might at formal functions, celebrations, or tailgates from ciders that might be consumed 
during dinner parties, lunch or dinner, with family, or during relaxation. The horseshoe shape of the initial consensus plot indicates that the sorting configuration probably requires a third dimension to adequately visualize (Leeuw, 2011; Nguyen \& Holmes, 2017). To better interpret the sorting configurations, a second consensus plot was generated with the second and third principal components. In the consensus plot of the second and third principal components (Figure 7), the second principal component separated ciders as it did in Figure 6 while the third principal component separates ciders panelists might consume at formal functions, parties, celebrations, and dinner parties from those that might be consumed during relaxation, with lunch or dinner, or at a bar. The third principal component acts much like the first but separates ciders panelists might consume at more casual or smaller events from those that might be consumed at more celebratory or larger gatherings.

Hierarchical clustering of dimensions 1,2, and 3 of the cross-product agreement matrix identified four distinct clusters. Cluster 1 (colored purple in Figures 6 and 7) contains ciders that panelists stated they would consume "any time", "at a bar", or at a "party." Ciders in this cluster $(\mathrm{C} 4, \mathrm{C} 5, \mathrm{C} 7, \mathrm{C} 12)$ are all found in metallic cans, something panelists may associate with more casual situations like parties or bar scenes, and casual consumption at any time. Cluster 2 (colored pink in Figure 6 and 7) contains ciders $(\mathrm{C} 9, \mathrm{C} 10, \mathrm{C} 14)$ that are found in 12 to 22 fluid ounce dark brown bottles with metallic caps and are also consumed in more casual occasions like "tailgating", "at home" (e.g., C9 and C10) or "with lunch or dinner" (e.g., C14). The ciders in clusters 1 and 2 share characteristics similar to that of beers, which may explain the casual occasions panelists might consume them in.

Cluster 3 (colored green in Figures 6 and 7) contains ciders panelists might consume at occasions they may deem more intimate or relaxed settings like "with family", "holidays". These 
ciders (C2, C6, C11) are all 750-ml large bottles in either clear or dark brown glass with simple renditions of animals on the label. The occasion they were sorted in indicates an association with seasonality, like summertime or cooler weather of the holidays, and shareability. The simplistic renditions of animals on the front labels might incline panelists to consume these ciders in more casual occasions. Cluster 4 (colored blue in Figures 6 and 7) contains ciders that panelists might consumer at larger get-togethers, like celebrations, with food, like lunch or dinner, or a combination of both, like a dinner party. These ciders (C1, C3, C8, C13, C15, C16, C17, C18) are bottled in dark green or brown bottles ranging from 500 to $750 \mathrm{ml}$ with either primarily red or white labels with a combination of delicate lettering and intricate drawings. The occasions these ciders were sorted into may indicate higher sophistication and shareability in more formal settings. Understanding what occasions panelists might consume a cider can help contextualize how much a consumer might be willing to pay for a cider and guide cider producers' choice on how they describe, market, and price their ciders.

\subsubsection{Comparison of Producer Descriptors and Panelist Expectations and Experiences}

There were clear patterns in how panelists sorted ciders based on their aroma, flavor, and packaging attributes. A comparison in how producers describe their ciders and what panelists expected and experienced showed numerous discrepancies (Table 1). The information generated from this study provides a first look into how well producers may set panelists' expectations as well as meeting them. "Cider" is abbreviated to "C" when discussing specific examples from these studies.

While there may be no surefire way of ensuring a product fully meets consumers' expectations, understanding how consumers may be perceiving a product through its packaging 
attributes could aid in the development of more representative labels and packaging that appropriately sets expectations while matching the producers' vision. When panelists visually evaluated ciders based on how they expected them to taste, there was little overlap between terms generated by panelists and those found on the label. A great number of ciders (e.g., C1, C3, C6, and C11) aim to connect through their front and back labels through descriptive paragraphs on their back label, but there was either little overlap or contrasting terminology. For example, $\mathrm{C} 8$ has a descriptive paragraph and dryness scale, but the majority of the descriptors provided were not used by panelists; instead, this cider was thought to be "fruity" and "sweet" rather than "fruity", "bright", "fresh" and "semi-dry". The solid red front label makes the dryness scale difficult to see and the small lettering on a white back label may have interfered with the panelist fully seeing what producers' have attempt to communicate. C6 describes its cider in a paragraph on its back label using 5 descriptive terms: dry, woody, full-bodied, astringent, and apple. Panelists mainly used two, "dry", which is found on the label, and "sweet", which is not. Creativity and individual expression can set regional and local producers apart but careful consideration of how consumers might perceive a product's label and packaging design is a necessary step in setting expectations and matching what is experienced.

Examples of ciders that were described by panelists using producers' terms in the visual evaluation of ciders are $\mathrm{C} 2$ and $\mathrm{C} 9 . \mathrm{C} 2$ has a label with contrasting colors, large letters and images, a descriptive paragraph of the cider's characteristics on the back label, and a large a dryness scale ("dry" as the leftmost anchor and "sweet" as the rightmost anchor) with the cider placed closer to the left. Another example of effective but non-verbal communication is found in C9. With a label containing warm colors, bees, and a name denoting sweetness, panelists mostly associated this cider with sweetness without the addition of descriptors or dryness scale. 
There was also little overlap in how producers described their ciders and what was actually experienced during the blinded sensory analysis of aroma and flavor. There were a number of ciders described as "dry", "semi-dry", or "off-dry" by producers that often described as "sweet" or "dry" with other sensory attributes like " flawed", " tart" or "bitter". Though the producers of C2 and C9 may have effectively conveyed their products' sensory attributes to panelists through its labels and packaging, what is actually experienced reveals a different narrative (Table 5). Instead of being described as "dry", "complex", and "tart" as they had in the visual evaluation, panelists commonly described C2 as "sweet" and "tart". A number of panelists described this cider as "sweet" during its visual examination, but this may be contributed to panelists who may have previously consumed the cider or based decisions without evaluating the back label. C9 was primarily described as "sweet" but panelists used additional terms such as "herbal", "flawed", and "fruity" to describe it, a trend that occurs for many ciders in this study. Cider producers may not be effectively communicating these terms on their packaging or label or there may be changes occurring in the product between when it is packaged, distributed, purchased, and consumed. Panelists also used "sweet" and "dry" when describing the same ciders (e.g., C4, C7, C11, C12, C13, C16). When cider is fermented to "dryness", it usually refers to the consumption and transformation of the majority sugars in a pre-fermentation mixture by yeasts to alcohol and $\mathrm{CO}_{2}$. A drying sensation may also be associated with tannin levels that some may refer to as "astringency" (A. G. H. Lea, 1995). How panelists and producers use of the terms "sweet" and "dry" may point to different understanding and usage among both cider producers', experts, and consumers with varying cider knowledge. 


\subsection{Conclusions and Future Work}

Though there is some overlap in how panelists sorted ciders based on their visual expectations of taste and the occasion they would most likely consume them in, there is little overlap in sorting configurations based on blind evaluation of aroma and flavor. These results indicate that Virginia ciders, when consumed, have distinct sensory attributes panelists could describe and categorize, but there are significant differences in how producers describe their product, how panelists expected ciders to taste, and what panelists actually experienced. In addition to differences between the descriptive language used by producers and panelists, there are opportunities for improved packaging types or monitoring of sensory changes that might occur after bottling, during distribution, or storage in retailers and after purchase in consumers' homes.

There are a number of ways to expand on this work. One advantage of DISTATIS is the generation of an assessor similarity matrix, which allows for examination of relationships between assessors. An atypical assessor or sets of assessors can be easily pulled from the consensus and analyzed separately (Abdi, Valentin, \& Ub, 2007). With a large number of assessors, cluster analysis can generate groups of assessors that sort ciders most similarly and then paired with demographic data to potentially explain if they share any commonalities. With a larger pool of panelists and use of a detailed demographic survey and questionnaire, further insight might be gained into who cider consumers are and the vocabulary they use based on factors such as age, alcoholic beverage consumption (wine versus beer drinkers versus nonalcoholic beverage consumers), and cultural or socioeconomic background.

Measurements of chemical parameters provided insight into the basic cider chemistry of samples, they were not good predictors of cider sorting and group labels. Using various gas 
chromatographic methods and HPLC analysis is recommended to measure residual sugars, titratable acidity (e.g.: malic, acetic, citric, lactic acids), sulfur-containing compounds (Guido \& Guido, 2016), glycerol, sorbitol, polyphenols, higher alcohols, and other volatile and non-volatile compounds. This will can provide a more reliable and fuller picture of Virginia ciders' chemistry and their respective sensory attributes. Testing protocols can be adapted from these findings and potentially help cider makers understand their cider's chemistry and what tests would be helpful in identifying chemical components that may be responsible for flaws or desirable attributes in their products.

Though providing photo sheets of ciders allowed for panelists to more easily sort and interact with a large sample set, having panelists physically interact and evaluate ciders might allow for results that are more representative when they are able to look at a product as they might in a retail environment. The results provide a first look as to how consumers sort cider through both visual and sensory analysis, but there is little insight into what drives liking, purchase intent, or willingness-to-pay. Affective and descriptive sensory testing, focus groups, and conjoint analysis are various methods that can be used to further expand on the knowledge gained through this study into something actionable for researchers, extension agents, or cider producers and experts. 


\section{References}

Abdi, H., Dunlop, J. P., \& Williams, L. J. (2009). How to compute reliability estimates and display confidence and tolerance intervals for pattern classifiers using the Bootstrap and 3-way multidimensional scaling (DISTATIS). NeuroImage, 45(1), 89-95. https://doi.org/10.1016/j.neuroimage.2008.11.008

Abdi, H., Valentin, D., Chollet, S., \& Chrea, C. (2007). Analyzing assessors and products in sorting tasks: DISTATIS, theory and applications. Food Quality and Preference, 18(4), 627-640. https://doi.org/10.1016/j.foodqual.2006.09.003

Abdi, H., Valentin, D., \& Ub, I. (2007). Some New and Easy Ways to Describe, Compare, and Evaluate Products and Assessors.

Anderson, H. (2002). Long Ashton Research Station: One hundred years of research. Pesticide Outlook, 13(5), 214-217. https://doi.org/10.1039/B209417G

Anton, M. J., Valles, B. S., Hevia, A. C., \& Lobo, A. P. (2014). Aromatic Profile of Ciders by Chemical Quantitative, Gas Chromatography-Olfactometry, and Sensory Analysis. Journal of Food Science, 79(1), S92-S99. https://doi.org/10.1111/1750-3841.12323

Beaton, D., Chin, C., \& Abdi, H. (2019). DiSTATIS Three Way Metric Multidimensional Scaling. Retrieved from https://cran.r-project.org/web/packages/DistatisR/DistatisR.pdf

De Queiroz, G., Hvitfeldt, E., Misra, K., Mastny, T., Erickson, J., Robinson, D., \& Silge, J. (2019). Text Mining for Word Processing and stemiment analysis using "dplyr", "ggplot2", and other tidy tools. Retrieved from https://cran.rproject.org/web/packages/tidytext/tidytext.pdf

Fabien-Ouellet, N., \& Conner, D. S. (2018). The Identity Crisis of Hard Cider. Journal of Food Research, 7(2), 54. https://doi.org/10.5539/jfr.v7n2p54 
GLINTCAP. (2019). Style Guidelines - Great Lakes International Cider and Perry Competition (GLINTCAP). Retrieved from https://glintcap.org/styles/

Hamza, V. K. (2014). A Study on the Mediation Role of Customer Satisfaction on Customer Impulse and Involvement to Word of Mouth and Repurchase Intention. International Journal of Business Insights \& Transformation. Retrieved from http://content.ebscohost.com/ContentServer.asp?T=P\&P=AN\&K=96263936\&S=R\&D=b th\&EbscoContent=dGJyMMTo50Sepq84zOX0OLCmr1Gep7NSsq24TbOWxWXS\&Co ntentCustomer=dGJyMPGsr1CyqbNNuePfgeyx43zx

Hopfer, H., \& Heymann, H. (2014). Judging wine quality: Do we need experts, consumers or trained panelists? Food Quality and Preference, 32, 221-233. https://doi.org/10.1016/j.foodqual.2013.10.004

Hout, M. C., Papesh, M. H., \& Goldinger, S. D. (2013). Multidimensional scaling. Wiley Interdisciplinary Reviews. Cognitive Science, 4(1), 93-103. https://doi.org/10.1002/wcs.1203

Lachowicz, S., Oszmiański, J., Uździcka, M., Chmielewska, J., Escuer, M. C., \& CampsBossacoma, M. (2019). The Influence of Yeast Strain, $\beta$-Cyclodextrin, and Storage Time on Concentrations of Phytochemical Components, Sensory Attributes, and Antioxidative Activity of Novel Red Apple Ciders. Molecules, 24(13), 2477. https://doi.org/10.3390/molecules24132477

Lahne, J., Abdi, H., \& Heymann, H. (2018). Rapid sensory profiles with DISTATIS and Barycentric Text Projection: An example with amari, bitter herbal liqueurs. Food Quality and Preference, 66, 36-43. https://doi.org/10.1016/j.foodqual.2018.01.003 
Lahne, J., Collins, T. S., \& Heymann, H. (2016). Replication Improves Sorting-Task Results Analyzed by DISTATIS in a Consumer Study of American Bourbon and Rye Whiskeys. Journal of Food Science, 81(5), S1263-S1271. https://doi.org/10.1111/1750-3841.13301

Lea, A. G. H. (1995). Cidermaking. In A. G. H. Lea \& J. R. Piggott (Eds.), Fermented Beverage Production (pp. 66-96). https://doi.org/10.1007/978-1-4757-5214-4_4

Lea, Andrew G. H., \& Arnold, G. M. (1978). The phenolics of ciders: Bitterness and astringency. Journal of the Science of Food and Agriculture, 29(5), 478-483. https://doi.org/10.1002/jsfa.2740290512

Leeuw, J. de. (2011). A Horseshoe for Multidimensional Scaling. Retrieved from https://escholarship.org/uc/item/67s0324c\#page=2

Lelièvre, M., Chollet, S., Abdi, H., \& Valentin, D. (2008). What is the validity of the sorting task for describing beers? A study using trained and untrained assessors. Food Quality and Preference, 19(8), 697-703. https://doi.org/10.1016/j.foodqual.2008.05.001

Mai, R., Symmank, C., \& Seeberg-Elverfeldt, B. (2016). Light and Pale Colors in Food Packaging: When Does This Package Cue Signal Superior Healthiness or Inferior Tastiness? Journal of Retailing, 92(4), 426-444. https://doi.org/10.1016/j.jretai.2016.08.002

Melcher, J. M., \& Schooler, J. W. (1996). The Misremembrance of Wines Past: Verbal and Perceptual Expertise Differentially Mediate Verbal Overshadowing of Taste Memory. Journal of Memory and Language, 35(2), 231-245. https://doi.org/10.1006/jmla.1996.0013 
Mueller, S., Osidacz, P., Francis, I. L., \& Lockshin, L. (2010). Combining discrete choice and informed sensory testing in a two-stage process: Can it predict wine market share? Food Quality and Preference, 21(7), 741-754. https://doi.org/10.1016/j.foodqual.2010.06.008

Nguyen, L. H., \& Holmes, S. (2017). Bayesian Unidimensional Scaling for visualizing uncertainty in high dimensional datasets with latent ordering of observations. $B M C$ Bioinformatics, 18(10), 65-79. https://doi.org/10.1186/s12859-017-1790-x

Nielsen. (2018). United States Association of Cider Makers and Nielsen Expand Their Analytic Relationship. Retrieved October 16, 2019, from https://www.nielsen.com/us/en/pressreleases/2018/united-states-association-of-cider-makers-and-nielsen-expand-relationsip

Nurin, T. (2018). Hard Cider Sales Slip And Then Rebound. Retrieved October 16, 2019, from Forbes website: https://www.forbes.com/sites/taranurin/2018/02/28/hard-cider-sales-slipthen-rebound/

Peng, B., Li, F., Cui, L., \& Guo, Y. (2015). Effects of Fermentation Temperature on Key Aroma Compounds and Sensory Properties of Apple Wine. Journal of Food Science, 80(12), S2937-S2943. https://doi.org/10.1111/1750-3841.13111

Peng, B., Yue, T., \& Yuan, Y. (2009). Analysis of key aroma components in cider from Shaanxi (China) Fuji apple. International Journal of Food Science \& Technology, 44(3), 610615. https://doi.org/10.1111/j.1365-2621.2008.01875.x

R Core Team. (2019). R: A language and environment for statistical computing. $R$ Foundation for Statistical Computing. Retrieved from https://www.R-project.org/.

Rinker, T. (2018). Tools for Stemming and Lemmatizing Text. Retrieved from https://cran.rproject.org/web/packages/textstem/index.html 
Sánchez, A., de Revel, G., Antalick, G., Herrero, Mó., García, L. A., \& Díaz, M. (2014). Influence of controlled inoculation of malolactic fermentation on the sensory properties of industrial cider. Journal of Industrial Microbiology \& Biotechnology.

Scott, J. A., \& Swaffield, C. H. (1998). Observations on the Influence of Temperature, Dissolved Oxygen and Juice Source on Stored Alcoholic Cider Flavour Development. Food Biotechnology, 12(1-2). https://doi.org/10.1080/08905439809549940

Spence, C., Wan, X., Woods, A., Velasco, C., Deng, J., Youssef, J., \& Deroy, O. (2015). On tasty colours and colourful tastes? Assessing, explaining, and utilizing crossmodal correspondences between colours and basic tastes. Flavour, 4(1), 23. https://doi.org/10.1186/s13411-015-0033-1

Symoneaux, R., Chollet, S., Patron, C., Bauduin, R., Le Quéré, J. M., \& Baron, A. (2015). Prediction of sensory characteristics of cider according to their biochemical composition: Use of a central composite design and external validation by cider professionals. $L W T$ Food Science and Technology, 61(1), 63-69. https://doi.org/10.1016/j.lwt.2014.11.030

Symoneaux, R., Guichard, H., Le Quéré, J. M., Baron, A., \& Chollet, S. (2015). Could cider aroma modify cider mouthfeel properties? Food Quality and Preference, 45, 11-17. https://doi.org/10.1016/j.foodqual.2015.04.004

The Cyder Market. (2019). Retrieved August 29, 2019, from https://www.cydermarket.com/ Thériault, F. (2019, February 20). Sector Trend Analysis - Cider market trends in the United States and the United Kingdom [Organizational description]. Retrieved October 16, 2019, from http://www.agr.gc.ca/eng/industry-markets-and-trade/international-agri-foodmarket-intelligence/reports/sector-trend-analysis-cider-market-trends-in-the-unitedstates-and-the-united-kingdom/?id=1549381895074 
USACM. (2018). United States Association of Cider Makers Style Guidelines. Retrieved from https://ciderassociation.org/wp-content/uploads/2018/12/USACM-Style-GuidelinesV2p0-1.pdf

Varela, P., \& Ares, G. (Eds.). (2014). Sorting Tasks. In Novel Techniques in Sensory Characterization and Consumer Profiling. https://doi.org/10.1201/b16853

Williams, A. A. (1974). Flavour Research and the Cider Industry. Journal of the Institute of Brewing, 80(5), 455-470. https://doi.org/10.1002/j.2050-0416.1974.tb06795.x

Williams, A. A., Lea, A. G. H., \& Timberlake, C. F. (1977). Measurement of Flavor Quality in Apples, Apple Juices, and Fermented Ciders. In R. A. Scanlan (Ed.), Flavor Quality: Objective Measurement (Vol. 51, pp. 71-88). https://doi.org/10.1021/bk-19770051.ch006

Williams, Anthony A. (1975). The development of a vocabulary and profile assessment method for evaluating the flavour contribution of cider and perry aroma constituents. Journal of the Science of Food and Agriculture, 26(5), 567-582.

https://doi.org/10.1002/jsfa.2740260503

Yu, X., Yuan, F., Fu, X., \& Zhu, D. (2016). Profiling and relationship of water-soluble sugar and protein compositions in soybean seeds. Food Chemistry, 196, 776-782. https://doi.org/10.1016/j.foodchem.2015.09.092 


\section{Chapter 4: Conclusions and Future Work}

Though there is some overlap in how panelists sorted ciders based on their expectations of taste and the occasion they would most likely consume them in, there is little overlap in sorting configurations based on blind evaluation of aroma and flavor. These results indicate that Virginia ciders, when consumed, have distinct sensory attributes consumers can describe and categorize, but there are marked differences in how producers describe their product, how panelists expected ciders to taste, and the sensory attributes panelists actually experienced. In addition to differences between the descriptive language used by producers and panelists, there are opportunities for improved packaging types or monitoring of sensory changes that might occur after bottling, during distribution, or storage in retailers and after purchase in consumers' homes. The results provide a first look as to how consumers sort cider through both visual and sensory evaluation, but there is little insight into what drives liking, purchase intent, or willingness-to-pay. Affective and descriptive sensory testing, focus groups, and conjoint analysis are various methods that can be used to further expand on the knowledge gained through this study into something actionable for researchers, extension agents, or cider producers and experts.

In this study, sorting tasks were successful in identifying categories of Virginia ciders and how consumers describe them. An advantage of DISTATIS is the generation of an assessor similarity matrix, which allows for examination of relationships between assessors. An atypical assessor or sets of assessors can be easily pulled from the consensus and analyzed separately (Abdi, Valentin, \& Ub, 2007). Performing sorting studies with a larger number of panelists, cluster analysis can be performed to identify groups of assessors that sort ciders most similarly. The demographic data of these panelists could then be evaluated to potentially explain if they share any commonalities. Further insight might be gained into who cider consumers are and 
identify segments of cider drinkers and the vocabulary they used based on factors such as age, alcoholic beverage consumption (wine versus beer drinkers versus non-alcoholic beverage consumption), and cultural or socioeconomic background. Sorting tasks can also be used to assess sensory changes in cider during distribution and storage and compare ciders produced in Virginia to other regional ciders to further define the US cider product space.

Sorting tasks are cost-effective, require little to no training, and produce multivariate data that can be statistically analyzed in a variety of ways but they do not provide insight into what drives sensory or product attributes drive liking, purchase intent, acceptance, or willingness-topay. Further hedonic or acceptance sensory evaluation is necessary to connect product liking and acceptance with the vocabulary used by consumers. For example, a cider that is described as "bitter" may be perceived have positive or negative connotations depending on the panelist's experiences and preferences. While inferences can be made if an attribute is associated with fermentation flaws in the literature, these do not provide a strong basis for commercial decisions. Check-all-that-apply (CATA) questionnaires are another rapid sensory analysis method that can incorporate hedonic responses with descriptive vocabulary.

In a CATA questionnaire, panelists are presented with a list of sensory attributes and asked to select the words or phrases that appropriately describe their experience with the product being evaluated. In addition to their ability to be combined with hedonic responses, the relationships between emotional responses, demographics, and purchase intention can be examined to provide insight into what drives liking (Meyners \& Castura, 2014). However, rapid sensory methods can be limited in their exploration of consumer attitudes and expectations of ciders and cannot be used alone to understand willingness-to-pay for a product as these decisions are made in absence of real situations (Lange, Martin, Chabanet, Combris, \& Issanchou, 2002). 
There are also a great number of factors that can influence an individual's choice in ciders. The beverage itself can be sparkling or still, dry or sweet, made with simply apples or a combination of fruits and spices. Cider can be packaged in a bottle or can, with varying sizes and labels. Where a cider is produced may also be of importance to the consumer with ciders coming from small-scale to commercial brewing companies. Focus groups are suggested for identifying differences in cider consumers' and what factors in Virginia ciders are important in their purchasing decision. They allow for detailed insight into consumer attitudes and the valuation of products and their respective attributes. Application in research with consumers of varying amounts of cider consumption can provide a framework of who these consumers are, what product attributes they value most, and how they respond to commercially available ciders. As there are few resources available on consumer knowledge, awareness, and usage of currently available cider style guides and descriptive terminology, these qualitative research methods allow for consumer conversations that generate rich contextual data that the previously mentioned methods cannot generate. This data collection method can also be one of the first steps in building surveys and identifying attributes for willingness-to-pay analysis.

Willingness-to-pay (WTP) is a measure of the price consumers will pay for a product containing specific attributes, including physical or sensory attributes that may enhance consumption or utility (Tozer, Galinato, Ross, Miles, \& McCluskey, 2015). Asking panelists to sort ciders into groups based on the occasions they might consume them in provided some insight into the occasions named and the cost of products consumed therein but how consumers value both sensory and visual attributes can be more accurately measured through WTP analysis, Hypothetical WTP analysis has been used in tandem with sensory analysis in studies in wine and beer such as the influence of beer pack type on WTP (Lefebvre \& Orlowski, 2019) or product 
information available on external packaging (Danner, Johnson, Ristic, Meiselman, \& Bastian, 2017). Only one study correlating hedonic liking and WTP has been conducted with cider and there are limited resources on the WTP of commercially available ciders (Tozer et al., 2015). Sensory analysis and traditional marketing research methodologies aim to tease out what a consumer truly wants and what they state they want, but certain methods can be costly, timeconsuming, or gather feedback that consumers think researchers want to hear. Conjoint analysis is another measure of hypothetical WTP and it is a "marketing technique for predicting how developed or redesigned products or services would perform when taken to market". Consumers are given pairs of products with varying combinations of attributes and price points and are asked to choose the one they prefer most (Hawley, 2008). Understanding how consumers value product attributes along with their hypothetical and non-hypothetical WTP can guide how consumers attribute value to a product's sensory or physical attributes but cannot fully capture consumer behavior when they are not under economic constraint. Experimental auctions measure non-hypothetical WTP by having consumers enter the experiment with the knowledge that they will be purchasing the product they are evaluating with their own money. Thus, it is in the consumer's best interest to submit a bid they are truly willing to pay based on their overall impression of the product. However, measurement of non-hypothetical WIP is limited in alcoholic beverages, potentially due to tight regulation in the US, but has been used to compare the WTP and product messaging during wine tourism (Eustice, McCole, \& Rutty, 2019).

Other limitations of this study are the provision of photo sheets rather than full-sized and unopened products. While photo sheets allowed for panelists to easily sort through a large number of products in a booth setting, photos cannot provide a complete visualization of the ciders' packaging and label. Due to the size of this study, space in the sensory laboratory was 
limited. Photo sheets of ciders allowed for panelists to more easily sort and interact with a large sample set, but having panelists physically interact and evaluate ciders might allow for results that are more representative when they are able to look at a product as they might in a retail environment. Evaluating products can be a multisensory experience of a product's texture, sound, weight, and color. Consumers interact with and choose products based on a wide array of factors and photos may have had panelists make sorting decisions in an unfamiliar fashion.

To improve on the reliability of the chemical analysis, a systematic approach of collecting and storing samples should be incorporated if the chemical analysis of the samples is to be performed. Rather than taking one sample from a single bottle, samples should be collected from a series of ciders of varying ages. The cider's production and bottling date and storage conditions such as light exposure, temperature, and time should be surveyed and collected for each sample wherever possible. Evidence of secondary fermentation should be noted. For this study, the reported chemical parameters include $\mathrm{pH}$, producer reported alcohol content, titratable acidity (measured as malic acid equivalents), and residual sugars (glucose and fructose) were measured. Though these measurements provided insight into the basic cider chemistry of samples, they were not good predictors of cider sorting and group labels. Using various gas chromatographic methods and HPLC analysis is recommended to measure residual sugars, titratable acidity (ex: malic, acetic, citric, lactic acids), sulfur-containing compounds (Guido \& Guido, 2016), glycerol, sorbitol, polyphenols, higher alcohols, and other volatile and non-volatile compounds. This will can provide a more reliable and fuller picture of Virginia ciders' chemistry and their respective sensory attributes. Testing protocols can be adapted from these findings and potentially help cider makers understand their cider's chemistry and what tests would be helpful 
in identifying chemical components that may be responsible for flaws or desirable attributes in their products. 


\section{References}

Abdi, H., Valentin, D., \& Ub, I. (2007). Some New and Easy Ways to Describe, Compare, and Evaluate Products and Assessors.

Danner, L., Johnson, T. E., Ristic, R., Meiselman, H. L., \& Bastian, S. E. P. (2017). "I like the sound of that!" Wine descriptions influence consumers' expectations, liking, emotions and willingness to pay for Australian white wines. Food Research International, 99, 263274. https://doi.org/10.1016/j.foodres.2017.05.019

Eustice, C., McCole, D., \& Rutty, M. (2019). The impact of different product messages on wine tourists' willingness to pay: A non-hypothetical experiment. Tourism Management, 72, 242-248. https://doi.org/10.1016/j.tourman.2018.11.022

Guido, L. F., \& Guido, L. F. (2016). Sulfites in beer: Reviewing regulation, analysis and role. Scientia Agricola, 73(2), 189-197. https://doi.org/10.1590/0103-9016-2015-0290

Hawley, S. T. (2008). Conjoint Analysis. The Patient: Patient-Centered Outcomes Research, 1(4), 255-257. https://doi.org/10.2165/1312067-200801040-00006

Lange, C., Martin, C., Chabanet, C., Combris, P., \& Issanchou, S. (2002). Impact of the information provided to consumers on their willingness to pay for Champagne: Comparison with hedonic scores. Food Quality and Preference, 13(7), 597-608. https://doi.org/10.1016/S0950-3293(02)00059-9

Lefebvre, S., \& Orlowski, M. (2019). Can, cup, or bottle? The influence of beverage vessel on taste and willingness to pay. International Journal of Hospitality Management, 76, 194205. https://doi.org/10.1016/j.ijhm.2018.05.009 
Meyners, M., \& Castura, J. C. (Eds.). (2014). Check-All-That-Apply Questions. In Novel Techniques in Sensory Characterization and Consumer Profiling (pp. 271-301). https://doi.org/10.1201/b16853

Tozer, P. R., Galinato, S. P., Ross, C. F., Miles, C. A., \& McCluskey, J. J. (2015). Sensory Analysis and Willingness to Pay for Craft Cider*. Journal of Wine Economics, 10(3), 314-328. https://doi.org/10.1017/jwe.2015.30 


\section{Figures, Tables, and Appendix \\ Figures}
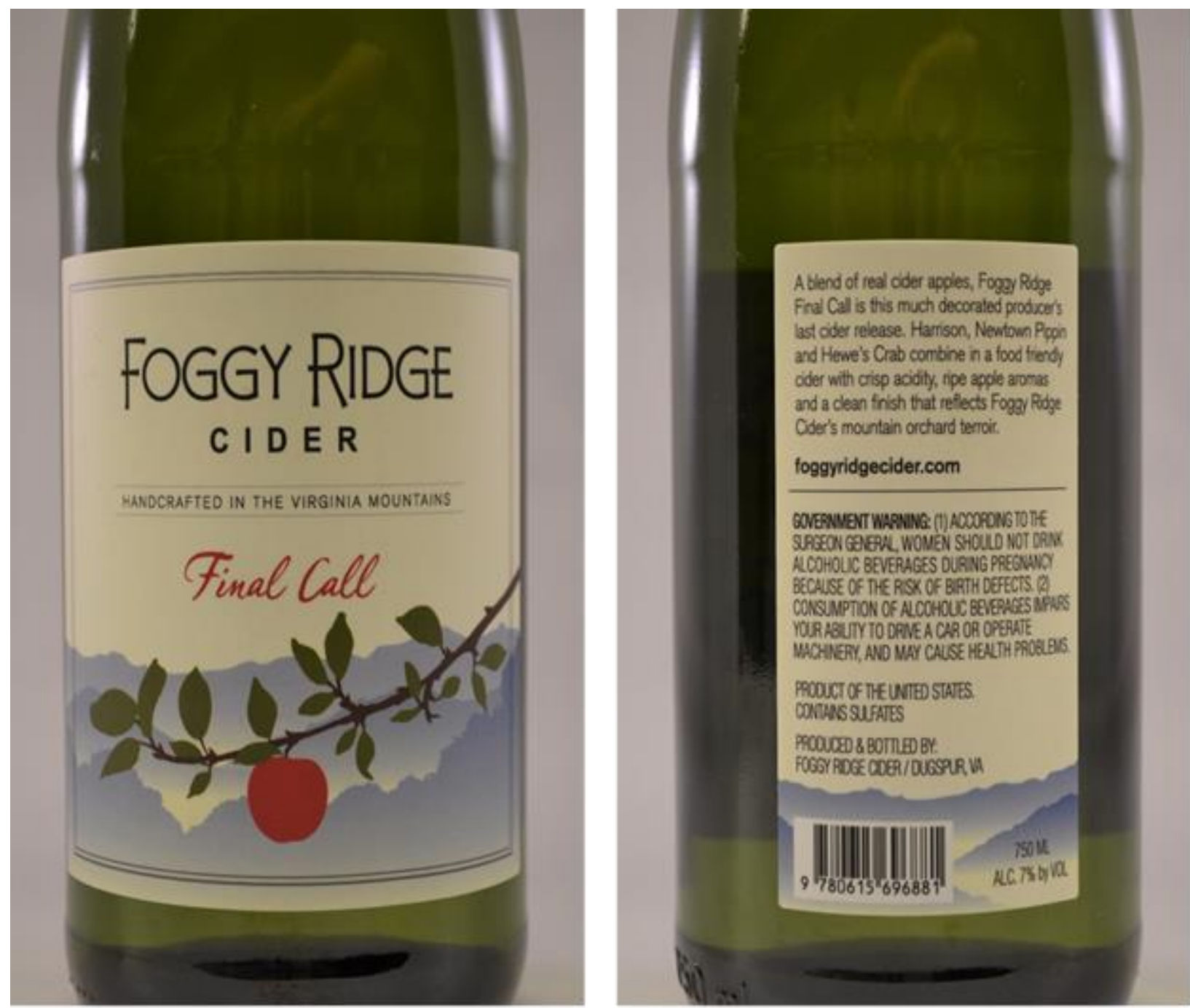

Figure 1. Examples of up-close photos of a cider sample included in the visual evaluation of cider packaging and label study. Panelists were able to visually examine the front and back labels of the cider packaging. 

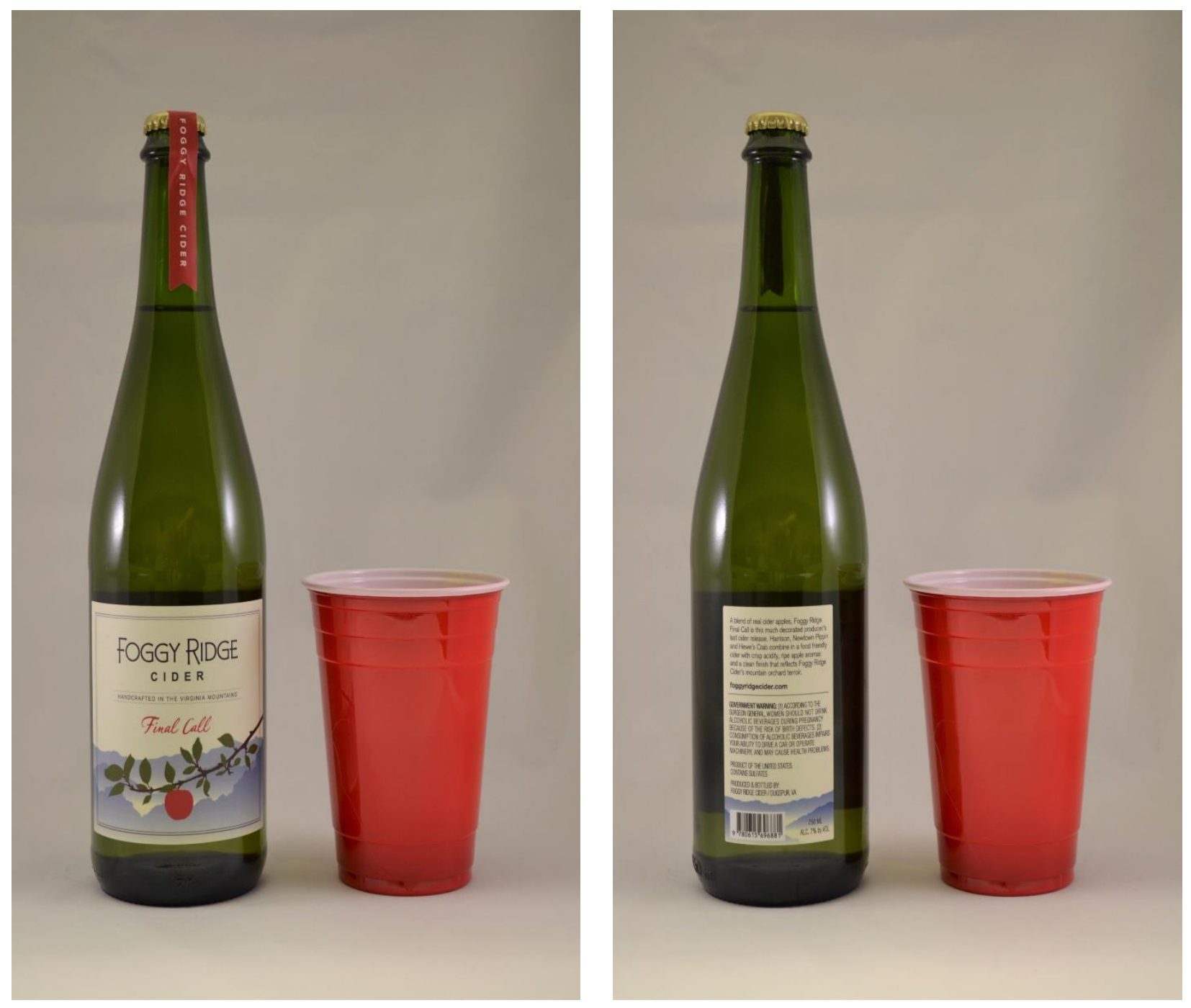

Figure 2. Examples of photos used to provide bottle/can size references to panelists. The ciders were intentionally photographed in a frame with a well-known reference item, an 18-ounce red plastic cup, to provide a size reference for panelists without them physically interacting with cider samples. 


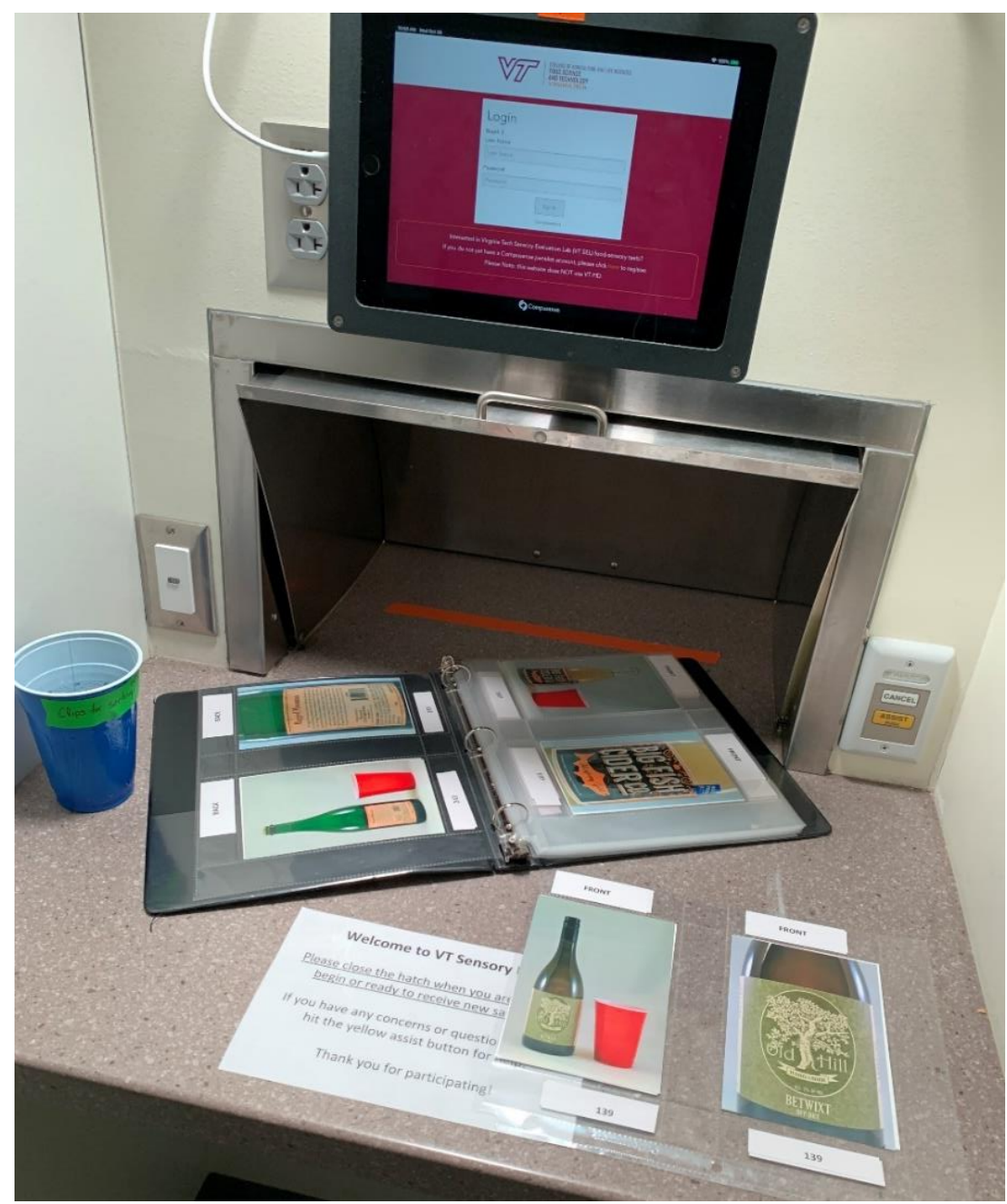

Figure 3. Example of in-booth set-up for the visual evaluation of packaging and label of the 18 ciders. Panelists were instructed by the prompts provided through Compusense on the pictured touch screen tablet. Each cider has a set of four photos illustrating the front and back of the product that were placed into one photo sheet labeled with a random 3-digit code. Two close-up photos (front and back of the packaging) were taken to showcase any verbiage, labels, photos, and other defining attributes of the packaging (manuscript, Figure 1). Two photos (front and back) were then taken of the full package next to an 18-ounce red plastic cup to demonstrate the size of the product (manuscript, Figure 2). Panelists were able to remove photo sheets during the sorting tasks and were provided with binder clips to aid in organization of groups. 


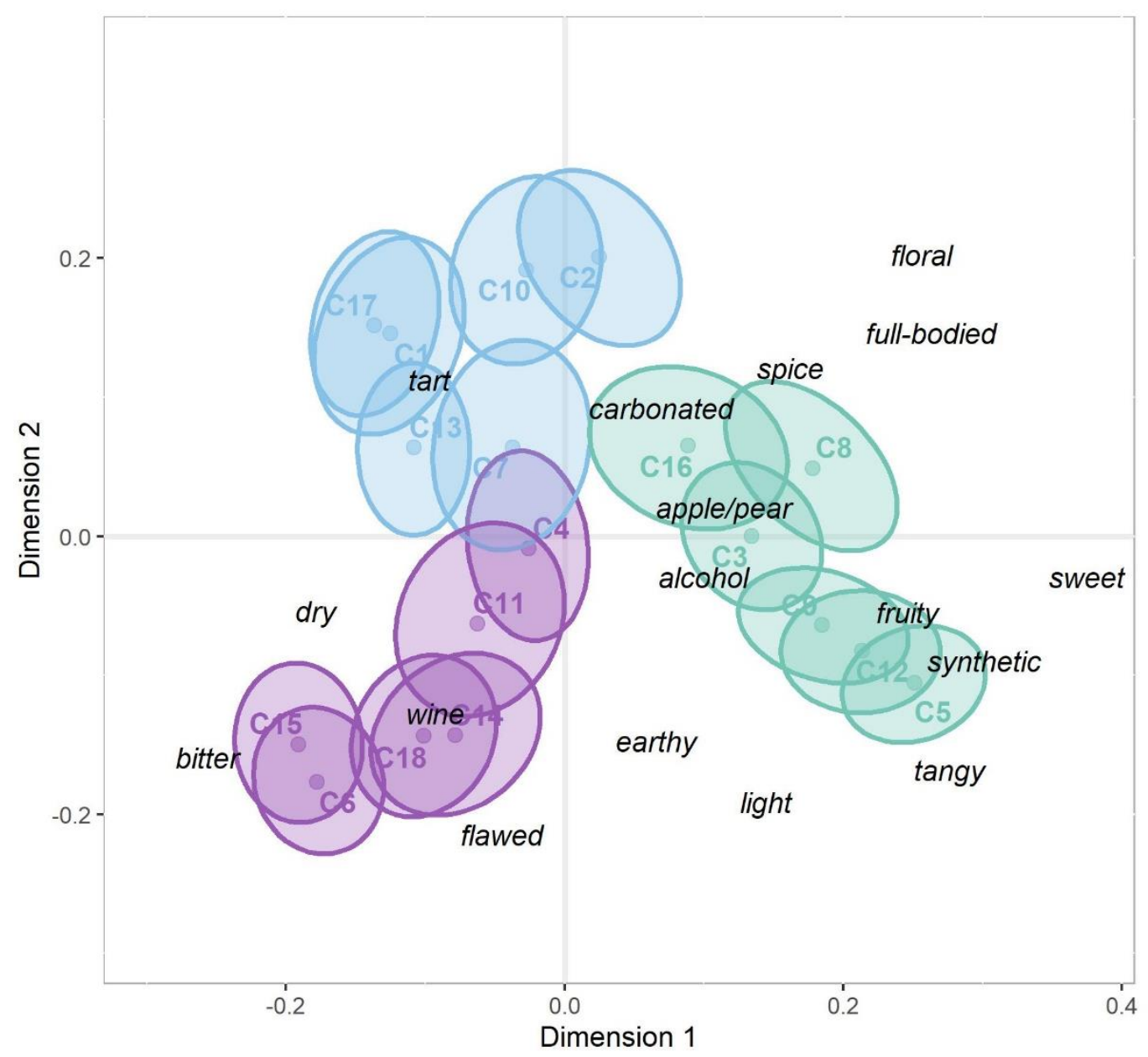

Figure 4. Aroma and flavor sorting study results generated by DISTATIS layered with categories generated from classical textual analysis. This 2-dimensional map illustrates the projection and placement of cider samples in space and the descriptor(s) that panelists most frequently used to describe them. The generated bootstrap confidence ellipses illustrate (with 95\% confidence) cider differences or similarities. Overlap of ellipses indicate similarities in cider sensory descriptors whereas no overlap is indicative of differences. The first dimension separated ciders which consumers labeled as "tart", "dry", "bitter", or "characterless" from those labeled as "herbal", "sweet", "tangy", "floral", and "fruity". The second principal component separated ciders labeled as "characterless", "funky", "light", "bitter", or "wine-like" from those labeled as "crisp", "floral", "tart", "carbonated", or "apple or pear-like". Hierarchical clustering of the first and second dimension of the cross-product agreement matrix revealed 3 distinct clusters of ciders: cluster 1 (colored blue) primarily consists of "tart" ciders, cluster 2 (colored purple) primarily consists of "dry" and "bitter" ciders, and cluster 3 (colored green) primarily consists of "sweet" and "fruity" ciders. 


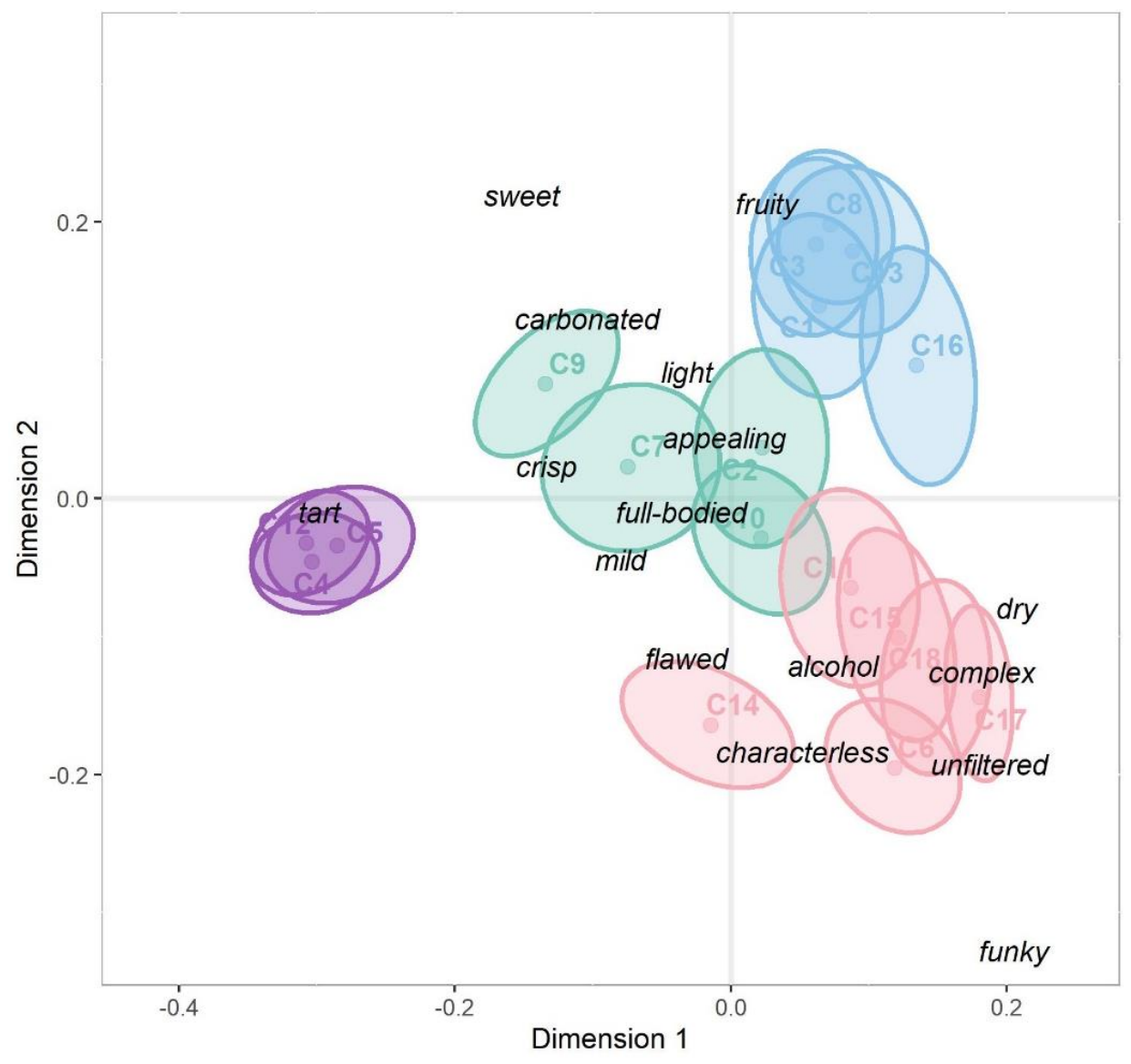

Figure 5. Visual evaluation of packaging and label results generated by DISTATIS that are based on how panelists expected they would taste, layered with categories generated from classical textual analysis. This 2-dimensional map illustrates the projection and placement of cider samples in space and the descriptor(s) that panelists most frequently used to describe them. The generated bootstrap confidence ellipses illustrate (with 95\% confidence) cider differences or similarities. Overlap of ellipses indicate similarities in cider sensory descriptors whereas no overlap is indicative of differences. The first dimension separated ciders panelists expected to taste "apple or pear-like", "tart", "sweet", or "carbonated" from those expected to be "tart", "earthy", "funky", "decadent", "dry", or "complex". The second principal component separated ciders expected to taste "bitter", "earthy", "barrel-aged", "funky", or "decadent" from those expected to be "sweet", "carbonated", "fruity", "light", or "crisp" from. Hierarchical clustering of dimensions 1 and 2 of the cross-product agreement matrix revealed 4 distinct clusters of ciders: cluster 1 (colored purple) contains ciders expected to be "tart", cluster 2 (colored green) contains ciders expected to be "crisp", "carbonated", or "light", cluster 3 (colored blue) contained ciders expected to be "fruity" or "light", and cluster 4 (colored pink) contains cider thought to be "complex", "unfiltered", "alcoholic", or "dry". 


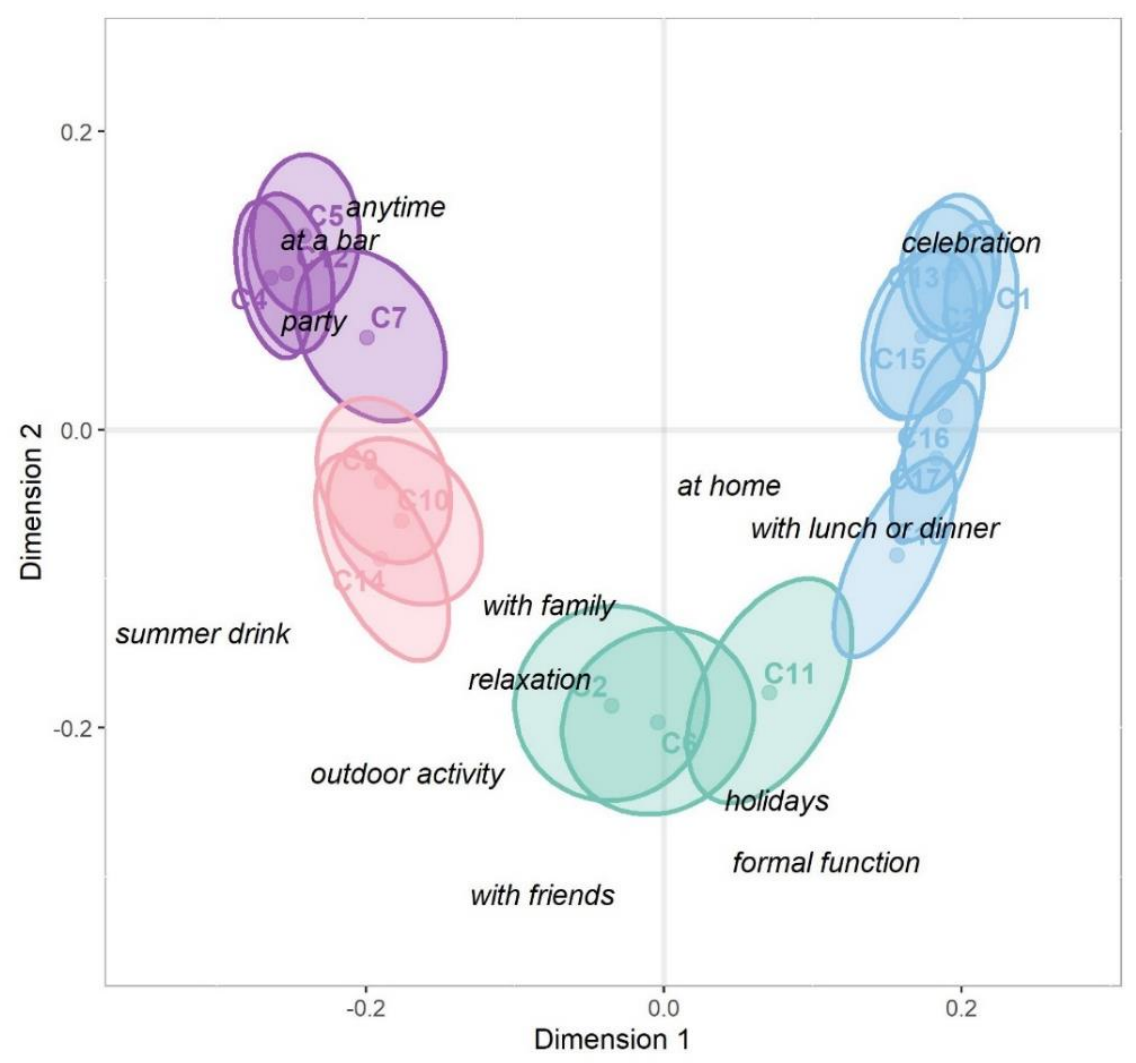

Figure 6. Visual evaluation of packaging and label results generated by DISTATIS that are based on the occasion panelists would consume them in, layered with categories generated from classical textual analysis. This 2-dimensional map illustrates the projection and placement of cider samples in space and the descriptor(s) that panelists most frequently used to describe them. The generated bootstrap confidence ellipses illustrate (with $95 \%$ confidence) cider differences or similarities. Overlap of ellipses indicate similarities in cider sensory descriptors whereas no overlap is indicative of differences. The first principal component separated ciders panelists might consume at "home", seasonal events during the summer or holidays, or with food from ciders that might be consumed at "tailgates", "dinner parties", "celebrations", or "at a bar". The second principal component separates ciders panelists might consume at "celebrations" and or "tailgates" from that might be consumed during "dinner parties", "with lunch or dinner", "with family", or during "relaxation". 


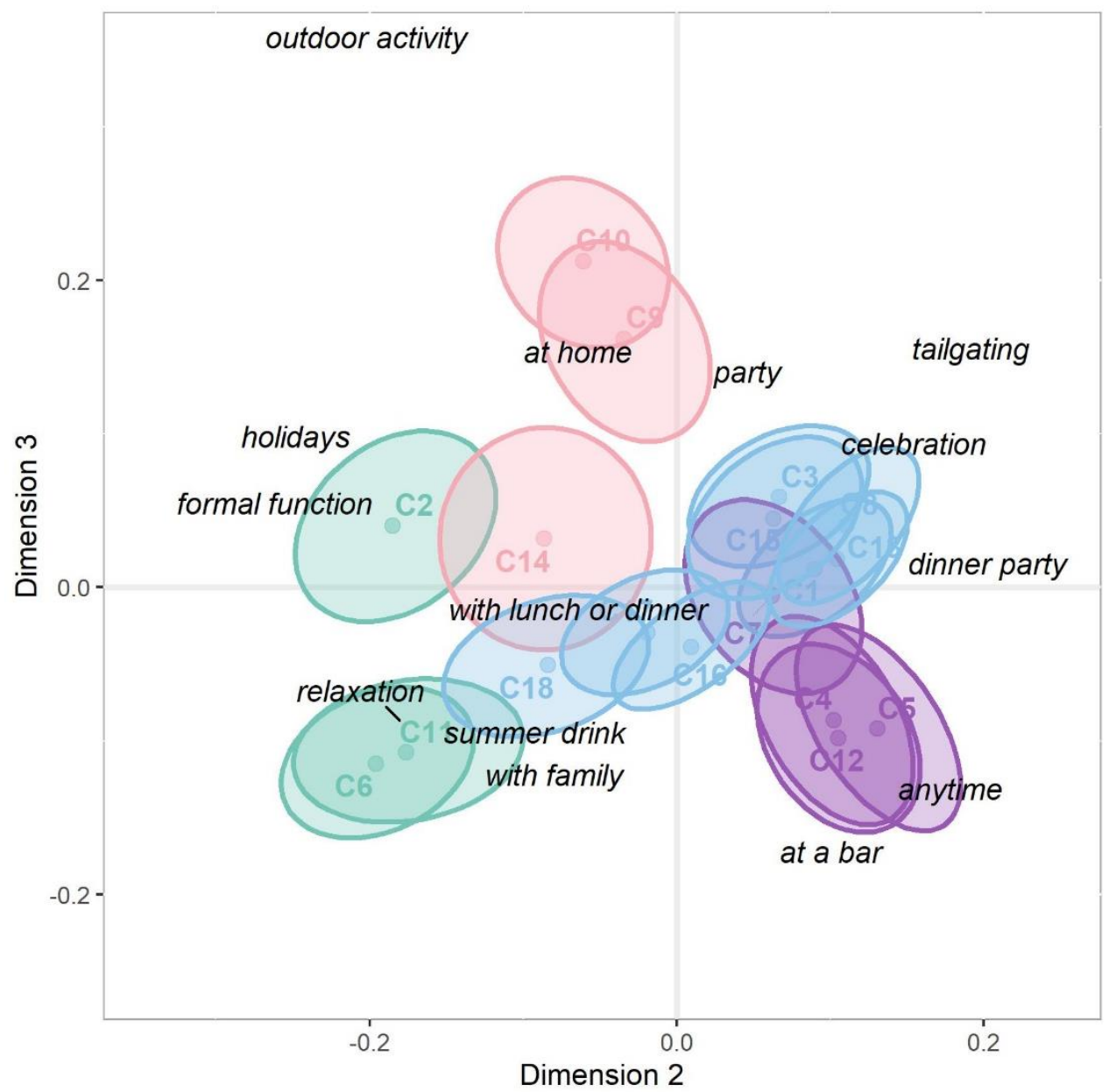

Figure 7. Visual evaluation of packaging and label results generated by DISTATIS that are based on the occasion panelists would consume them in, layered with categories generated from classical textual analysis. This 2-dimensional map illustrates the projection and placement of cider samples in space and the descriptor(s) that panelists most frequently used to describe them. The generated bootstrap confidence ellipses illustrate (with 95\% confidence) cider differences or similarities. Overlap of ellipses indicate similarities in cider sensory descriptors whereas no overlap is indicative of differences. The second principal component separates ciders panelists might at "formal functions", "celebrations", or "tailgates" from ciders that might be consumed during "dinner parties", "with lunch or dinner", "with family", or "relaxation". The third principal component separates ciders panelists might consume at "formal functions", "parties", "celebrations", and "dinner parties" from those that might be consumed during "relaxation", "with lunch or dinner", or "at a bar". 


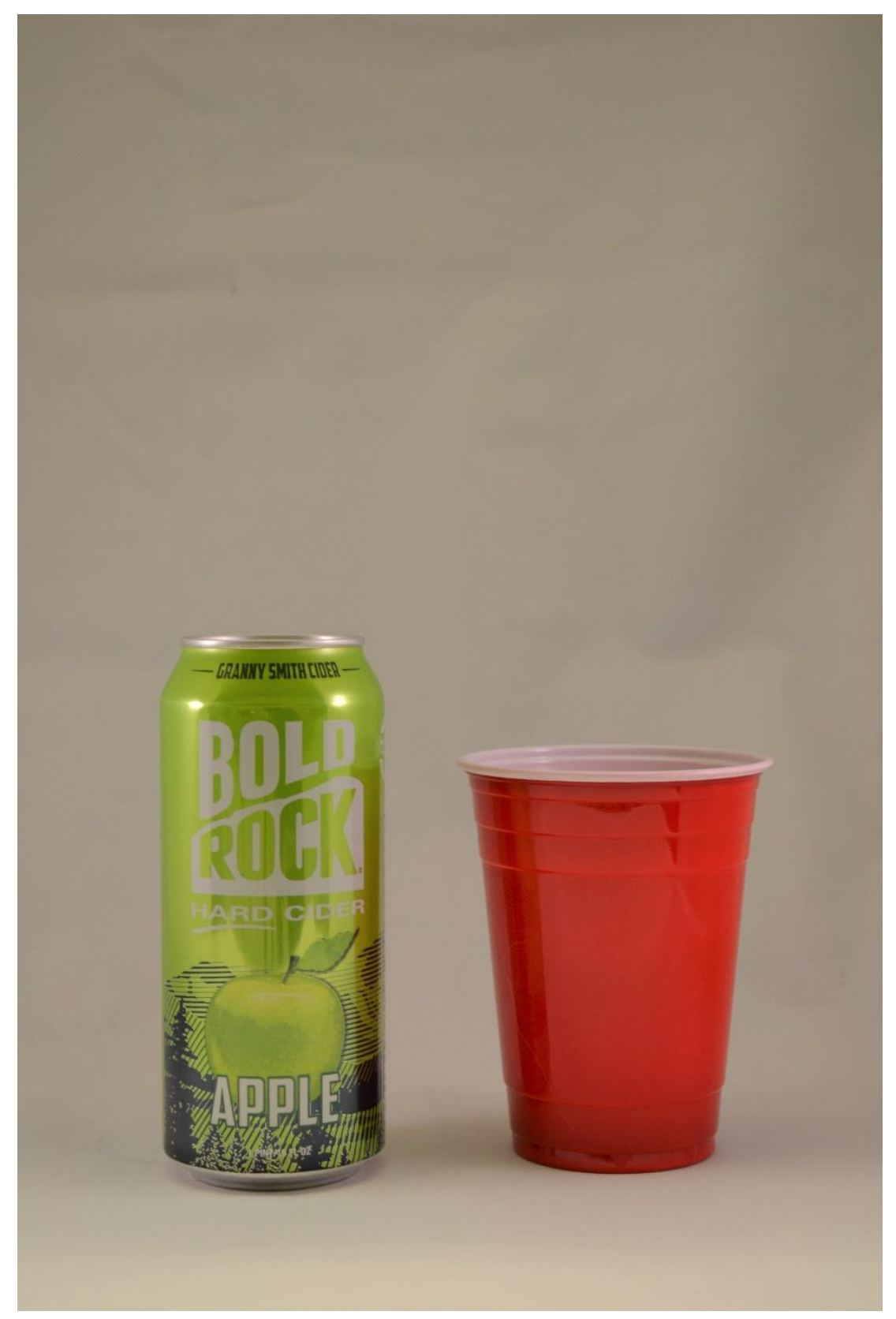

Figure 8. An example of a cider that panelists expected to taste "tart" when sorting in the visual evaluation of external product attributes. 


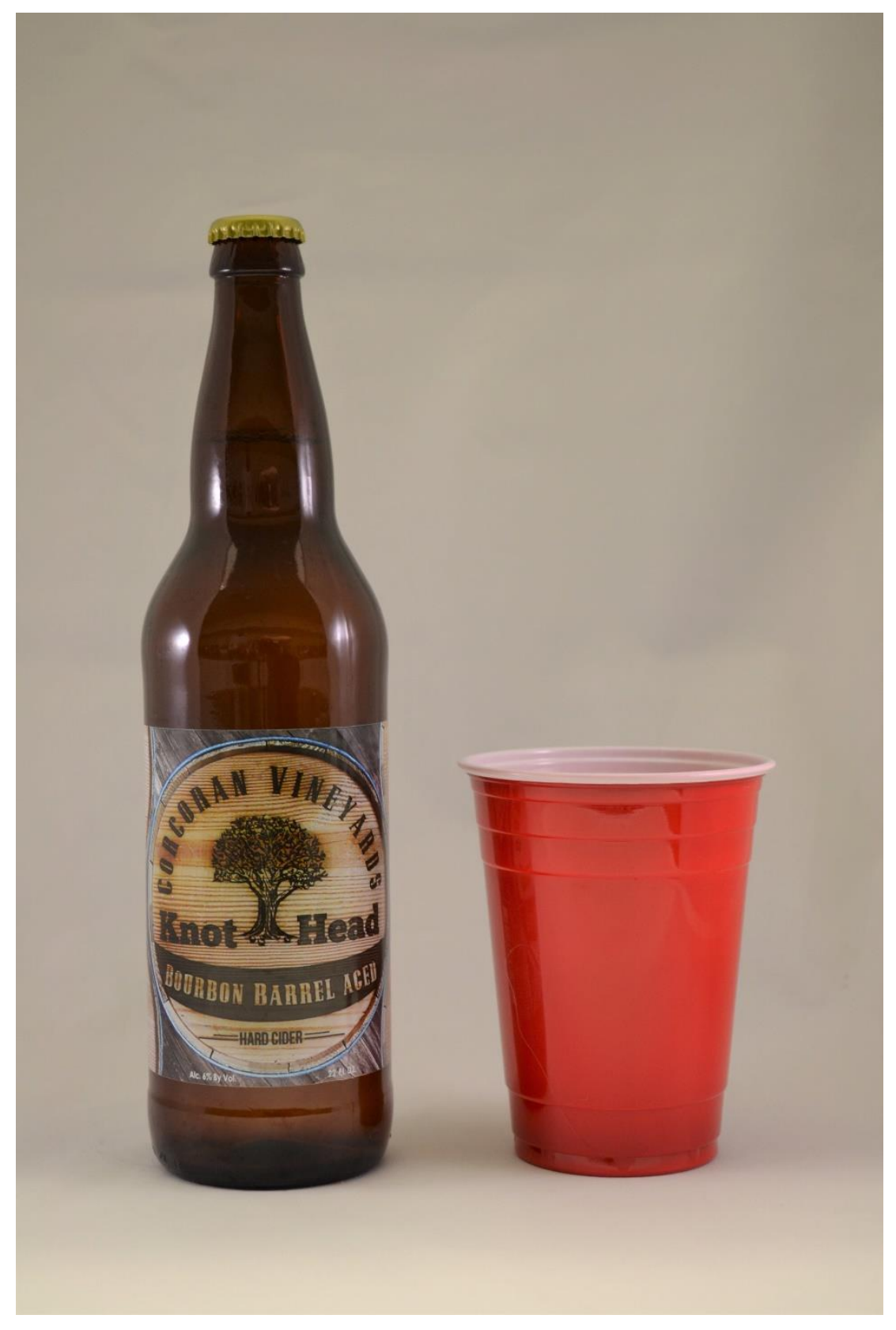

Figure 9. An example of a cider that panelists expected to taste "carbonated" or "full-bodied" when sorting in the visual evaluation of external product attributes. 


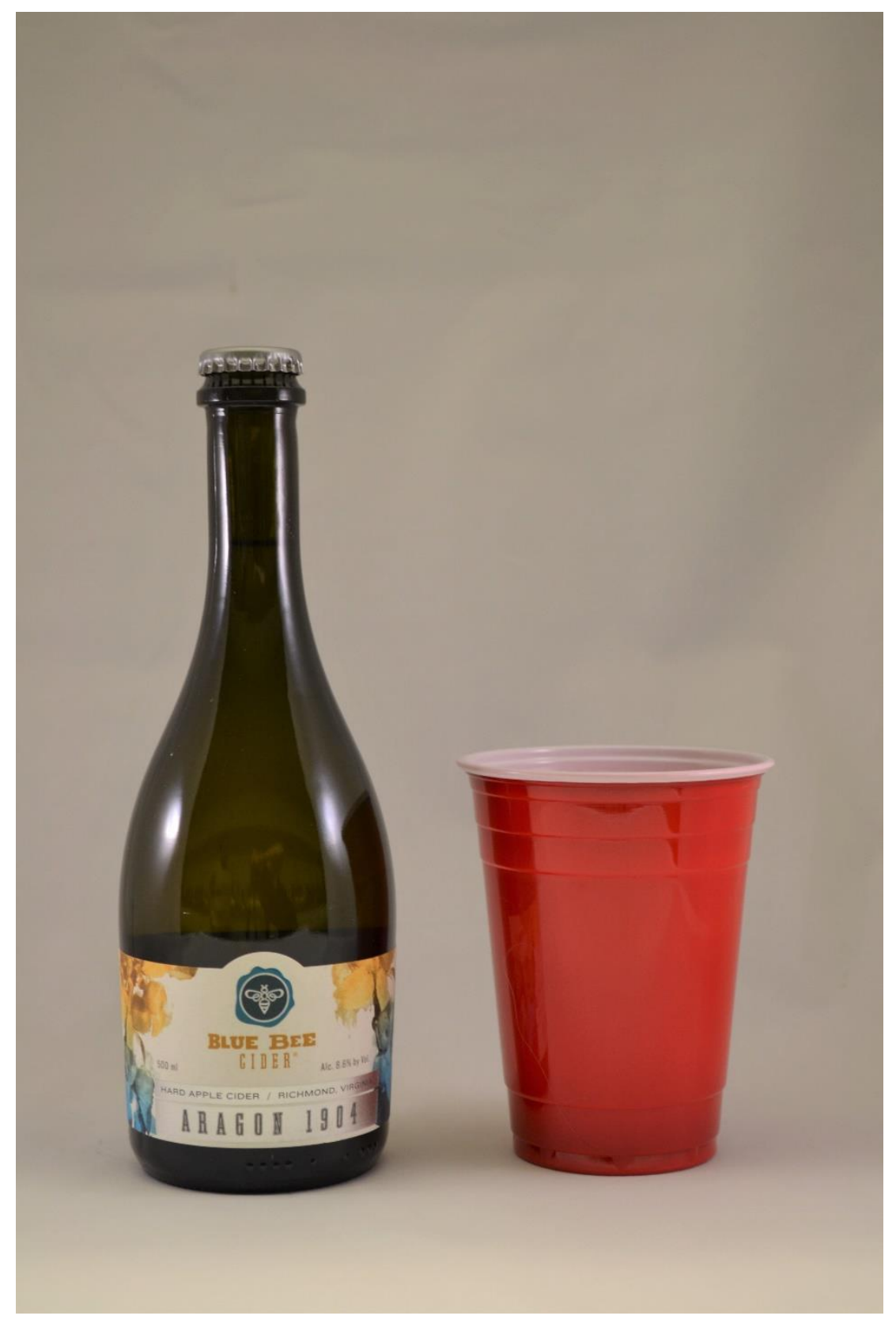

Figure 10. An example of a cider that panelists expected to taste "fruity" or "light" when sorting in the visual evaluation of external product attributes. 


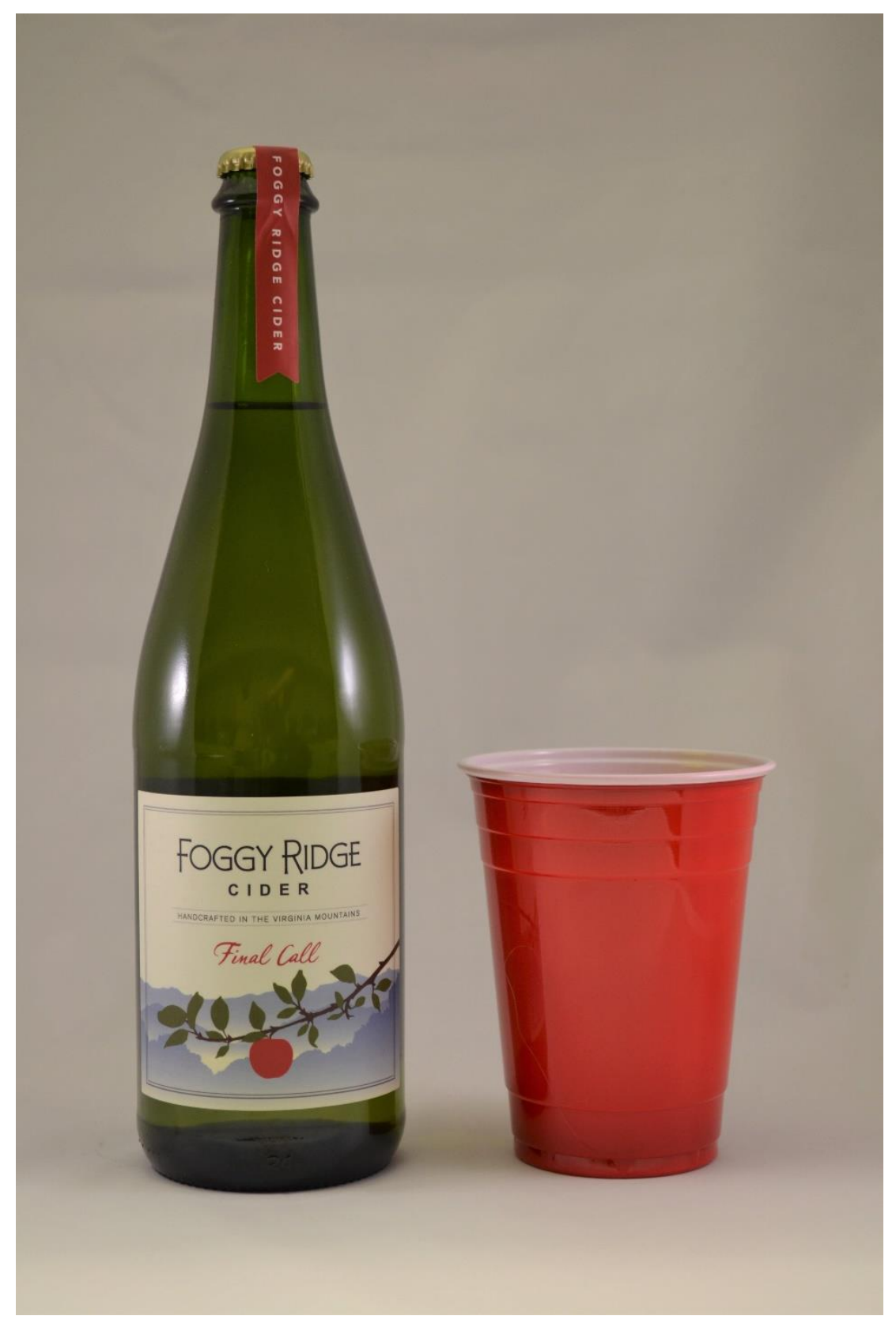

Figure 11. An example of a cider that panelists expected to taste "alcoholic", "complex", or "unfiltered" when sorting in the visual evaluation of external product attributes. 


\section{Tables}

Table 1. Tabulated list of cider packaging and labels such as size, packaging color and type, prominent label colors, and images.

\begin{tabular}{|c|c|c|c|c|}
\hline Cider (C) & Size & $\begin{array}{c}\text { Packaging Color } \\
\text { And Type } \\
\end{array}$ & $\begin{array}{c}\text { Primary Label } \\
\text { Color }\end{array}$ & Label Images \\
\hline $\mathrm{C} 1$ & $750 \mathrm{~mL}$ & $\begin{array}{l}\text { Dark green glass } \\
\text { with metallic cap }\end{array}$ & $\begin{array}{c}\text { Beige, yellows, } \\
\text { green }\end{array}$ & Animal \\
\hline $\mathrm{C} 2$ & $750 \mathrm{~mL}$ & $\begin{array}{l}\text { Clear glass with } \\
\text { metallic cap }\end{array}$ & Black and yellow & Apple \\
\hline $\mathrm{C} 3$ & $500 \mathrm{~mL}$ & $\begin{array}{l}\text { Dark green bottle } \\
\text { with metallic cap }\end{array}$ & $\begin{array}{l}\text { White, yellow, and } \\
\text { blue }\end{array}$ & $\begin{array}{c}\text { Apple tree and } \\
\text { gentleman with hat }\end{array}$ \\
\hline $\mathrm{C} 4$ & 16 fluid ounces & Metallic can & $\begin{array}{l}\text { White, green, and } \\
\text { blue }\end{array}$ & Apple \\
\hline $\mathrm{C} 5$ & 16 fluid ounces & Metallic can & Green and white & Insect \\
\hline C6 & $750 \mathrm{~mL}$ & $\begin{array}{c}\text { Dark brown glass } \\
\text { with cork }\end{array}$ & Blue and red & Tree etched in wood \\
\hline $\mathrm{C} 7$ & 12 fluid ounces & Metallic can & $\begin{array}{c}\text { White, red, yellow, } \\
\text { and black }\end{array}$ & $\begin{array}{c}\text { Farmhouse and } \\
\text { animal }\end{array}$ \\
\hline $\mathrm{C} 8$ & $500 \mathrm{~mL}$ & $\begin{array}{l}\text { Dark green bottle } \\
\text { with metallic cap }\end{array}$ & Red and white & Animal \\
\hline C9 & 12 fluid ounces & $\begin{array}{l}\text { Dark brown bottle } \\
\text { with metallic cap }\end{array}$ & Orange and red & Animal \\
\hline $\mathrm{C} 10$ & 22 fluid ounces & $\begin{array}{l}\text { Dark brown glass } \\
\text { with metallic cap }\end{array}$ & Blue and brown & Animal \\
\hline $\mathrm{C} 11$ & $750 \mathrm{~mL}$ & $\begin{array}{c}\text { Clear glass with } \\
\text { swing top }\end{array}$ & White and purple & Apple on branch \\
\hline $\mathrm{C} 12$ & 16 fluid ounces & Metallic can & Green and silver & $\begin{array}{c}\text { Insect and floral } \\
\text { shapes }\end{array}$ \\
\hline $\mathrm{C} 13$ & $750 \mathrm{~mL}$ & $\begin{array}{l}\text { Dark green glass } \\
\text { with metallic cap }\end{array}$ & White and blue & Star \\
\hline $\mathrm{C} 14$ & 22 fluid ounces & $\begin{array}{l}\text { Dark brown glass } \\
\text { with metallic cap }\end{array}$ & $\begin{array}{c}\text { Green, yellow, blue, } \\
\text { brown }\end{array}$ & Apple on branch \\
\hline $\mathrm{C} 15$ & $750 \mathrm{~mL}$ & $\begin{array}{l}\text { Dark green glass } \\
\text { with metallic cap }\end{array}$ & Red and gold & Apple \\
\hline $\mathrm{C} 16$ & $750 \mathrm{~mL}$ & $\begin{array}{c}\text { Dark green glass } \\
\text { with metallic } \\
\text { screw top } \\
\end{array}$ & Green and white & Tree \\
\hline $\mathrm{C} 17$ & $750 \mathrm{~mL}$ & $\begin{array}{c}\text { Dark brown glass } \\
\text { with cork }\end{array}$ & Brown and beige & Sketch of apple tree \\
\hline $\mathrm{C} 18$ & $750 \mathrm{~mL}$ & $\begin{array}{l}\text { Dark brown glass } \\
\text { with metallic cap }\end{array}$ & White, red, brown & Animal \\
\hline
\end{tabular}


Table 2. Overview of panelist $(N=65)$ demographics highlighting gender, age, how often they purchased hard cider, and their consumption of other alcoholic beverages other than cider.

\begin{tabular}{c|c|c} 
Variable & Description & Frequency $(\boldsymbol{N})$ \\
\hline \multirow{3}{*}{ Gender } & Male & 47 \\
& Female & 17 \\
& Other & 1 \\
\hline \multirow{3}{*}{ Age Range (years) } & $21-30$ years & 32 \\
& $31-40$ years & 16 \\
& $41-50$ years & 11 \\
Cider Purchase Frequency & $51-60$ years & 2 \\
\hline & $61-70$ years & 8 \\
& Less than once a month & 38 \\
Other Alcoholic Beverage & $1-4$ times a month & 15 \\
Consumption & Once a week & 4 \\
\hline & More than once a week & 60 \\
& Wine & 46 \\
& Beer & 11
\end{tabular}


Table 3. Categories of cider attributes that panelists $(N=60)$ reported were the most important, appealing, or influential in their decision to purchase cider. Column 1 contains examples of attribute listed by panelists, column 2 contains the category in which they were placed, and column 3 demonstrates the frequency this category occurred.

\begin{tabular}{|c|c|c|}
\hline Example of Attribute & Category & Frequency $(n)$ \\
\hline $\begin{array}{c}\text { Sweetness } \\
\text { Fruity } \\
\text { Refreshing } \\
\text { Flavor } \\
\text { Apple flavor } \\
\text { Dry } \\
\text { Funky } \\
\text { Taste } \\
\text { Flavor profile and intensity } \\
\text { Carbonation } \\
\text { Aroma }\end{array}$ & $\begin{array}{c}\text { Anticipated or experienced sensory } \\
\text { attributes }\end{array}$ & 48 \\
\hline $\begin{array}{c}\text { Price } \\
\text { Cost } \\
\text { Economic }\end{array}$ & Cost & 10 \\
\hline $\begin{array}{c}\text { Locality } \\
\text { Brewery Location } \\
\text { Origin }\end{array}$ & Location Produced & 9 \\
\hline $\begin{array}{c}\text { Cider style } \\
\text { Style } \\
\text { Alcohol content } \\
\end{array}$ & Cider Style & 6 \\
\hline $\begin{array}{c}\text { Apple type } \\
\text { Apple variety } \\
\text { Pears } \\
\text { Pineapple }\end{array}$ & Apple or fruit variety & 6 \\
\hline $\begin{array}{c}\text { Familiarity } \\
\text { New } \\
\text { Recognition }\end{array}$ & Newly or previously experienced & 6 \\
\hline Brand & Brand & 5 \\
\hline Label & Label & 4 \\
\hline
\end{tabular}


Table 4. Chemical composition of cider samples. Mean values and the range of standard deviation are reported for $\mathrm{pH}$, titratable acidity, and residual sugars (glucose, fructose, and total). The acid-sugar ratio has been calculated to illustrate balance between residual sugars and titratable acidity.

\begin{tabular}{|c|c|c|c|c|c|c|c|}
\hline \multirow{2}{*}{$\begin{array}{l}\text { Cider } \\
\text { (C) }\end{array}$} & \multirow{2}{*}{ pH } & \multirow{2}{*}{$\begin{array}{c}\text { Ethanol } \\
(\%)\end{array}$} & \multirow{2}{*}{$\begin{array}{c}\text { Titratable } \\
\text { Acidity } \\
\text { (g/L) }\end{array}$} & \multicolumn{3}{|c|}{ Residual Sugars } & \multirow{2}{*}{$\underset{(\%)}{\text { Acid/Sugar Ratio }}$} \\
\hline & & & & Fructose $(\mathrm{g} / \mathrm{L})$ & Glucose $(\mathrm{g} / \mathrm{L})$ & $\begin{array}{l}\text { Total } \\
(\mathrm{g} / \mathrm{L})\end{array}$ & \\
\hline C1 & $3.47 \pm 0.01^{\mathrm{i}}$ & 7.70 & $2.46 \pm 0.10^{\mathrm{de}}$ & $2.94 \pm 0.22^{\mathrm{efg}}$ & $5.00 \pm 0.45^{\mathrm{bcd}}$ & $7.93 \pm 0.62^{\text {def }}$ & $31.09^{\text {cde }}$ \\
\hline $\mathrm{C} 2$ & $3.48 \pm 0.01^{\mathrm{i}}$ & 8.20 & $2.46 \pm 0.10^{\mathrm{de}}$ & $3.97 \pm 0.88^{\operatorname{def}}$ & $6.00 \pm 1.41^{b c d}$ & $9.96 \pm 2.29^{\text {bcdef }}$ & $25.35^{\text {cde }}$ \\
\hline $\mathbf{C 3}$ & $3.56 \pm 0.01^{\mathrm{g}}$ & 8.60 & $1.97 \pm 0.10 \mathrm{f}^{\mathrm{g}}$ & $5.66 \pm 0.82^{\text {bcde }}$ & $8.87 \pm 1.32^{b}$ & $14.53 \pm 2.13^{\mathrm{bcd}}$ & $13.72^{\mathrm{e}}$ \\
\hline $\mathrm{C4}$ & $3.47 \pm 0.00^{\mathrm{i}}$ & 5.50 & $1.88 \pm 0.07^{\mathrm{g}}$ & $5.64 \pm 1.21^{\text {bcde }}$ & $5.41 \pm 1.22^{\mathrm{bcd}}$ & $11.05 \pm 2.43^{\text {bcde }}$ & $17.72^{\mathrm{de}}$ \\
\hline $\mathrm{C5}$ & $3.55 \pm 0.17^{\mathrm{g}}$ & 4.70 & $1.45 \pm 0.10^{\mathrm{h}}$ & $8.55 \pm 0.22^{a b c}$ & $8.60 \pm 0.94^{\mathrm{b}}$ & $17.16 \pm 1.13^{b c}$ & $8.50^{\mathrm{e}}$ \\
\hline C6 & $3.84 \pm 0.01^{\mathrm{d}}$ & 7.90 & $0.98 \pm 0.08^{\mathrm{i}}$ & $0.00 \pm 0.00^{\mathrm{g}}$ & $1.99 \pm 0.51^{\mathrm{d}}$ & $1.99 \pm 0.51^{\mathrm{f}}$ & $51.75^{\mathrm{bc}}$ \\
\hline C7 & $3.69 \pm 0.01^{\mathrm{e}}$ & 5.50 & $1.45 \pm 0.04^{\mathrm{h}}$ & $7.30 \pm 1.18^{\mathrm{abcd}}$ & $6.32 \pm 0.91^{\mathrm{bcd}}$ & $13.62 \pm 2.09^{\mathrm{bcd}}$ & $10.81^{\mathrm{e}}$ \\
\hline C8 & $3.50 \pm 0.01^{h}$ & 6.30 & $2.50 \pm 0.10^{\mathrm{d}}$ & $10.58 \pm 3.27^{\mathrm{a}}$ & $7.10 \pm 2.18^{\mathrm{bc}}$ & $17.68 \pm 5.46^{\mathrm{b}}$ & $14.86^{\mathrm{e}}$ \\
\hline C9 & $3.52 \pm 0.01^{h}$ & 6.80 & $2.79 \pm 0.10^{\mathrm{c}}$ & $9.68 \pm 2.39^{\mathrm{a}}$ & $19.18 \pm 4.51^{\mathrm{a}}$ & $28.86 \pm 6.90^{\mathrm{a}}$ & $10.02^{\mathrm{e}}$ \\
\hline C10 & $3.44 \pm 0.00^{\mathrm{j}}$ & 6.00 & $2.55 \pm 0.07^{\mathrm{cd}}$ & $4.38 \pm 0.65^{\mathrm{def}}$ & $4.78 \pm 0.71^{\mathrm{bcd}}$ & $9.16 \pm 9.16^{\text {cdef }}$ & $28.17^{\text {cde }}$ \\
\hline C11 & $3.67 \pm 0.01^{\mathrm{f}}$ & 7.50 & $2.19 \pm 0.04^{\text {ef }}$ & $0.00 \pm 0.00^{\mathrm{g}}$ & $4.75 \pm 1.31^{\mathrm{bcd}}$ & $4.75 \pm 1.31^{\text {ef }}$ & $48.17^{\mathrm{c}}$ \\
\hline $\mathrm{C} 12$ & $3.85 \pm 0.00^{\mathrm{d}}$ & 6.90 & $3.39 \pm 0.01^{\mathrm{a}}$ & $8.78 \pm 2.35^{\mathrm{ab}}$ & $6.56 \pm 1.82^{\mathrm{bcd}}$ & $15.34 \pm 4.17^{\mathrm{bcd}}$ & $23.11^{\text {cde }}$ \\
\hline $\mathrm{C} 13$ & $3.45 \pm 0.01^{\mathrm{j}}$ & 7.00 & $3.11 \pm 0.08^{b}$ & $4.73 \pm 1.39^{\text {cdef }}$ & $5.03 \pm 1.47^{\mathrm{bcd}}$ & $9.76 \pm 2.86^{\text {bcdef }}$ & $33.37^{\text {cde }}$ \\
\hline C14 & $3.95 \pm 0.01^{\mathrm{a}}$ & 5.50 & $1.25 \pm 0.19^{\mathrm{hi}}$ & $1.37 \pm 0.48^{\mathrm{fg}}$ & $3.07 \pm 0.95^{\mathrm{cd}}$ & $4.44 \pm 1.43^{\text {ef }}$ & $29.12^{\text {cde }}$ \\
\hline C15 & $3.89 \pm 0.00^{\mathrm{c}}$ & 7.20 & $1.45 \pm 0.04^{\mathrm{h}}$ & $0.00 \pm 0.00^{\mathrm{g}}$ & $1.87 \pm 0.36^{\mathrm{d}}$ & $1.87 \pm 0.36^{f}$ & $79.45^{\mathrm{b}}$ \\
\hline C16 & $3.66 \pm 0.00^{f}$ & 7.00 & $1.86 \pm 0.04^{\mathrm{g}}$ & $5.42 \pm 1.07^{\text {bcde }}$ & $7.12 \pm 1.35^{\mathrm{bc}}$ & $12.54 \pm 2.41^{\text {bcde }}$ & $15.16^{\mathrm{e}}$ \\
\hline C17 & $3.47 \pm 0.01^{\mathrm{i}}$ & 8.50 & $2.10 \pm 0.04^{\mathrm{fg}}$ & $0.00 \pm 0.00^{\mathrm{g}}$ & $1.80 \pm 0.37^{\mathrm{d}}$ & $1.80 \pm 0.37^{\mathrm{f}}$ & $120.65^{\mathrm{a}}$ \\
\hline C18 & $3.92 \pm 0.00^{b}$ & 7.00 & $1.86 \pm 0.10^{\mathrm{g}}$ & $0.00 \pm 0.00^{\mathrm{g}}$ & $4.20 \pm 1.01^{\mathrm{bcd}}$ & $4.20 \pm 1.01^{\text {ef }}$ & $46.32^{\mathrm{cd}}$ \\
\hline
\end{tabular}

*Ethanol as reported by producers on label

** Calculated as malic acid equivalents

*** Percentage calculated by dividing titratable acidity by total residual sugars 
Table 5. Comparison of cidermaker descriptors and panelist descriptors in both the visual and sensory evaluation of ciders. From left to right: Column 1 contains blinded ciders, column 2 is a tabulates producer descriptors of the cider, column 3 contains expected sensory attributes $(n>$ $5)$, and column 4 contains experienced sensory attributes of blinded ciders $(n>5)$.

\begin{tabular}{|c|c|c|c|}
\hline $\begin{array}{c}\text { Cider } \\
(\mathbf{C})\end{array}$ & Label Descriptors & Expected Sensory Attributes & $\begin{array}{c}\text { Experienced Sensory } \\
\text { Attributes }\end{array}$ \\
\hline $\mathrm{C} 1$ & Semi-dry & Dry, sweet & Dry, flawed, tart, bitter, \\
\hline $\mathrm{C} 2$ & $\begin{array}{l}\text { Complex, bright, apple, } \\
\text { fruity, semi-dry }\end{array}$ & Dry, sweet, complex, tart & Sweet, tart \\
\hline $\mathrm{C} 3$ & Light, crisp, off-dry & Dry, sweet & Sweet, apple/pear, fruity \\
\hline $\mathrm{C} 4$ & Refreshing, tart, clean & Tart, sweet & Sweet, dry, flawed \\
\hline $\mathrm{C} 5$ & Refreshing, crisp & Apple, sweet, tart, crisp & Sweet \\
\hline C6 & $\begin{array}{l}\text { Dry, woody, full- } \\
\text { bodied, astringent, } \\
\text { apple }\end{array}$ & Dry, sweet & $\begin{array}{l}\text { Tart, dry, bitter, } \\
\text { characterless }\end{array}$ \\
\hline $\mathrm{C} 7$ & None & Sweet & Sweet, dry \\
\hline $\mathrm{C} 8$ & $\begin{array}{l}\text { Bright, fresh, fruity, } \\
\text { semi-dry }\end{array}$ & Fruity, sweet & $\begin{array}{l}\text { Sweet, tart, apple/pear, } \\
\text { fruity }\end{array}$ \\
\hline C9 & Fruity, sweet & Sweet & $\begin{array}{l}\text { Sweet, herbal, flawed, } \\
\text { fruity }\end{array}$ \\
\hline $\mathrm{C} 10$ & $\begin{array}{l}\text { Barrel-aged, refreshing, } \\
\text { crisp }\end{array}$ & Dry, sweet, complex & Sweet, tart \\
\hline $\mathrm{C} 11$ & $\begin{array}{l}\text { Dry, carbonated, barrel- } \\
\text { aged }\end{array}$ & Dry, tart & $\begin{array}{l}\text { Tart, dry, fruity, bitter, } \\
\text { flawed, sweet }\end{array}$ \\
\hline $\mathrm{C} 12$ & Crisp, clean & Sweet, tart, crisp & Sweet, flawed, light, fruity \\
\hline $\mathrm{C} 13$ & Semi-dry & Dry, sweet, tart, crisp & Sweet, dry \\
\hline C14 & Dry & Dry, sweet & Sweet, tart \\
\hline $\mathrm{C} 15$ & Off-dry & Dry, sweet & Dry, flawed, bitter \\
\hline $\mathrm{C} 16$ & $\begin{array}{c}\text { Carbonated, fruity, dry, } \\
\text { crisp, tart, bright, } \\
\text { balanced, refreshing }\end{array}$ & Dry, sweet & $\begin{array}{l}\text { Sweet, fruity, apple/pear, } \\
\text { dry }\end{array}$ \\
\hline $\mathrm{C} 17$ & Dry & Dry, tart, crisp & Tart, dry, bitter \\
\hline $\mathrm{C} 18$ & Dry & Dry & Dry, flawed, funky, tart \\
\hline
\end{tabular}




\section{Appendix}

Appendix 1. Screening survey used to identify panelists for the free-sorting task of Virginia hard ciders

1. Are you above 21 years of age? *Please note, if you are under 21 we cannot accept your participation due to IRB protocol. You will be required to present a valid Government Issued ID before the start of any alcohol-related tests.

a. Yes

b. No (REMOVE)

2. Do you consume alcoholic beverages?

a. Yes

b. No (REMOVE)

3. Have you ever had hard cider?

a. Yes

b. No

4. Is there any reason you cannot consume alcohol?

a. Yes (REMOVE)

b. No

5. Do you have allergies to any of the following? (Check all that apply)

a. Alcohol or other fermented beverages (REMOVE)

b. Apples and/or pears (REMOVE)

c. None of the above 
Appendix 2. Free-sorting prompt seen by panelists in the free sorting study using sensory evaluation of Virginia hard ciders.

Please read the following instructions carefully before you begin:

You have eighteen (18) samples of Virginia hard ciders in front of you in labeled glasses. Please smell and taste all samples and make groups based on their similarities.

- You can use any criteria you want to sort these samples into groups

- You can make any number of groups between two (2) and seventeen (17).

- You can put as many samples as you like into each group.

- After sorting, please write a few words for the "label" to describe that group of samples.

- If you use multiple terms or phrases to describe a group, please separate the terms or phrases by semicolons (;). 
Appendix 3. Demographic questionnaire and survey used to catalogue panelist' demographics and alcohol purchase and consumption habits.

Thank you for completing the first section of this test. You will now be asked to complete a brief questionnaire containing both demographic and study-related questions. Please ensure you answer all questions before exiting the test.

1. Please indicate your gender:
a. Male
b. Female
c. Other: (Please indicate)

2. Please indicate your age:
a. 21-30 years old
b. 31-40 years old
c. 41-50 years old
d. 51-60 years old
e. 61-70 years old
f. 71-80 years old
g. 81 years old or above

3. How often do you purchase hard cider?
a. I do not purchase hard cider
b. Once a month or less
c. Once every two weeks
d. Once a week
e. More than once a week

4. What other alcoholic beverages do you consume? (Check all that apply)
a. I only consume hard cider
b. Wine (red, white, sparkling, etc.)
c. Wine coolers
d. Beer
e. Spirits (whiskey, bourbon, vodka, rum, gin, etc.
f. Liqueurs (fruit, flower, cream, honey, etc.)
g. Other(s): please indicate

5. When purchasing hard cider, what is most (important/appealing/influential) to you? Please insert "N/A" you do not consume hard cider.
a. (Free response)
b. N/A for non-cider drinkers

6. How often do you typically consume hard cider?
a. Never
b. Less than 1 time per month
c. 1-4 times a month
d. Once per week
e. More than once per week 
Appendix 4. Free-sorting prompt used to guide the evaluation of visual attributes of Virginia hard ciders based on perceptions of taste and occasions they would be consumed by panelists. Prompts were presented in randomized order.

You have eighteen (18) photos of Virginia hard ciders in front of you.

One side of each sheet has 2 photographs of the cider's front label, and the other side has 2 photographs of the cider's back label.

Taste and occasion sorting prompts, alternated in order presentation:

Taste Prompt:

Please evaluate the front and back labels of all 18 hard ciders and sort them into groups according to how you think the hard ciders will taste.

- After sorting, please write a few words for the "label" to describe that group of samples.

- If you use multiple terms or phrases to describe a group, please separate the terms or phrases by semicolons (;).

- You can make any number of groups between two (2) and seventeen (17).

- You can put as many samples as you like into each group.

\section{Occasion prompt:}

Please evaluate the front and back labels of all 18 hard ciders and sort them into groups according to what OCCASION you would be most inclined to drink them at.

- After sorting, please write a few words for the "label" to describe that group of samples.

- If you use multiple terms or phrases to describe a group, please separate the terms or phrases by semicolons (;).

- You can make any number of groups between two (2) and seventeen (17).

- You can put as many samples as you like into each group. 


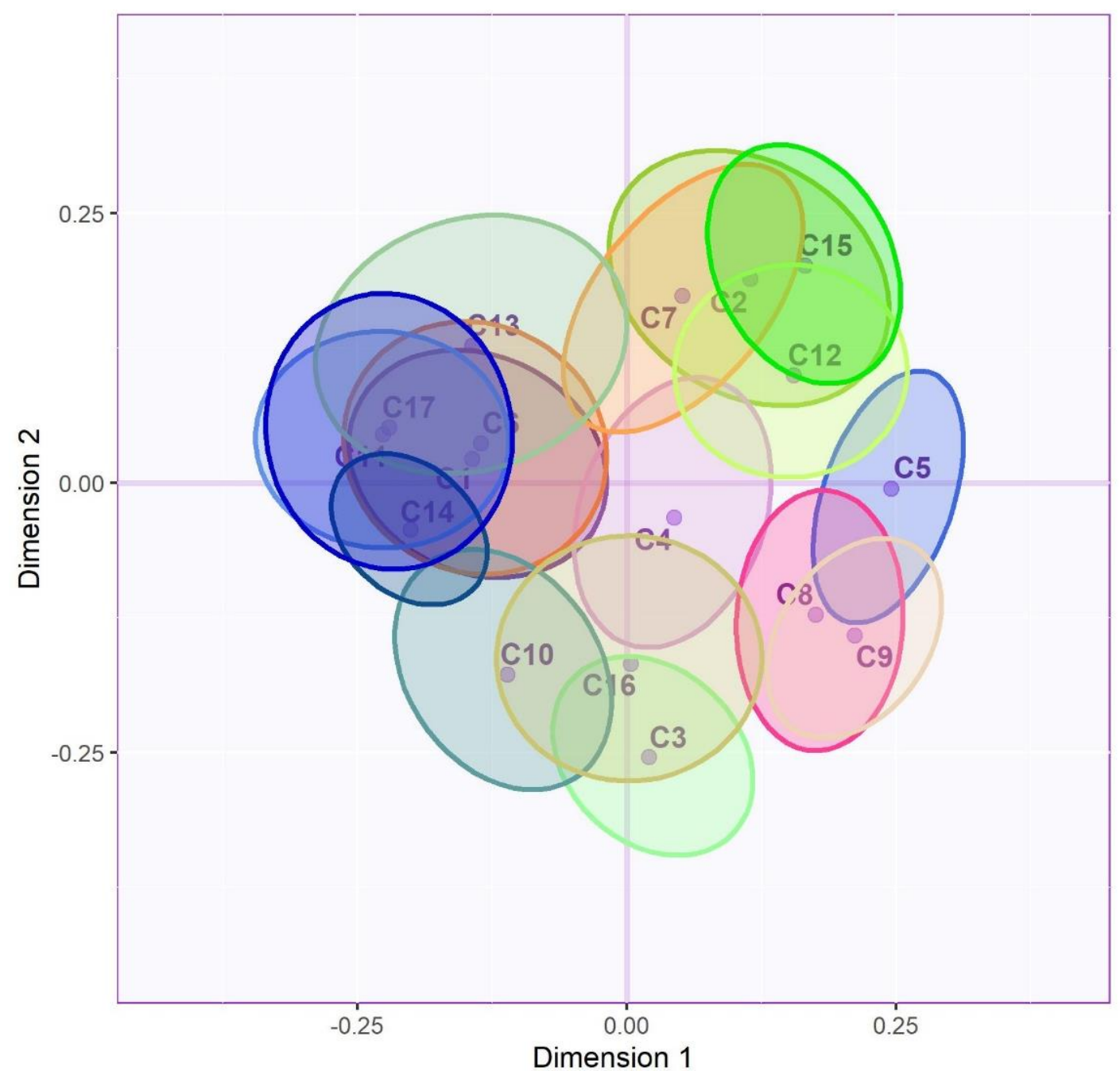

Appendix Figure 1. Sensory sorting study results generated by aroma and flavor evaluation of $\left(\mathrm{K}_{1}=18\right)$ from omitted panelists $\left(\mathrm{N}_{1}=16\right)$ This 2-dimensional map illustrates the projection and placement of cider samples in space. The generated bootstrap confidence ellipses illustrate (with 95\% confidence) cider differences or similarities. Overlap of ellipses indicate product similarities in whereas no overlap is indicative of differences. 